\title{
The Gas Disk: Evolution and Chemistry
}

\author{
Christian $\operatorname{Rab}^{1}$ (D) C Carla Baldovin-Saavedra ${ }^{1}$. \\ Odysseas Dionatos $^{1} \cdot$ Eduard Vorobyov $^{1,2}$. \\ Manuel Güdel ${ }^{1}$
}

Received: 12 January 2016 / Accepted: 28 November 2016 / Published online: 7 December 2016

(C) The Author(s) 2016. This article is published with open access at Springerlink.com

\begin{abstract}
Protoplanetary disks are the birthplaces of planetary systems. The evolution of the star-disk system and the disk chemical composition determines the initial conditions for planet formation. Therefore a comprehensive understanding of the main physical and chemical processes in disks is crucial for our understanding of planet formation. We give an overview of the early evolution of disks, discuss the importance of the stellar high-energy radiation for disk evolution and describe the general thermal and chemical structure of disks. Finally we provide an overview of observational tracers of the gas component and disk winds.
\end{abstract}

Keywords Stars: pre-main sequence - Stars: formation - Protoplanetary disks - Accretion, accretion disks $\cdot$ Planet-disk interactions $\cdot$ ISM: jets and outflow $\cdot$ Astrochemistry

\section{Introduction}

Disks around young stellar objects are the birthplaces of planetary systems. A comprehensive knowledge of their evolution, structure and chemical composition is therefore crucial for our understanding of planet formation.

Low mass stars like our Sun are formed with a disk component. Observations show that the fraction of disk-bearing stars in clusters with an age of $\approx 1 \mathrm{Myr}$ is usually $\gtrsim 80 \%$. However, the disk fraction drops quite rapidly with cluster age. In clusters with ages of $2-3 \mathrm{Myr}$ still $\approx 50 \%$ of the stars show disks, whereas in older $(\approx 10 \mathrm{Myr})$ clusters the fraction of disk-bearing stars is lower than $5 \%$ (e.g. Hernández et al. 2008; Fedele et al. 2010). This tells us that disks have a typical lifetime of a few million years. The lifetime of disks defines the timescale on which formation of massive (gaseous) planets can occur.

C. Rab

christian.rab@univie.ac.at

1 Department of Astrophysics, University of Vienna, Türkenschanzstr. 17, 1180 Vienna, Austria

2 Research Institute of Physics, Southern Federal University, Stachki 194, Rostov-on-Don, 344090, Russia 
Disks evolve on timescales of a few million years. The evolutionary stages are determined by the balance of accretion onto the disk and different disk dispersal/removal mechanisms (see Williams and Cieza 2011 for a review). There is observational evidence that disks form already within $10^{4}-10^{5} \mathrm{yr}$ after the gravitational collapse of the parental cloudcore (e.g. Murillo et al. 2013) at the phase where the star is still deeply embedded (Class-0 protostellar phase; see review of Li et al. 2014). After the formation disks are growing due to the continuous inflow of mass from their surrounding envelopes during the Class- 0 and Class-I (the star becomes visible) protostellar phases. Once this reservoir is drained and the star reaches its Class-II phase ( $\mathrm{T}$ Tauri star), disk erosion processes due to accretion onto the star, disk photoevaporation, jet acceleration, and planet formation lead to the disappearance of dust and gas disks within typically 2-3 Myr (although in some rare cases disks are still present at ages of $\sim 10 \mathrm{Myr}$ ). Observations of disks with large inner holes/gaps, the so called transitional disks, indicate that disk dispersal works from inside out. From the fraction of observed transition disks a timescale of $\gtrsim 10^{5} \mathrm{yr}$ is derived for this period (see Espaillat et al. 2014 for a review). The final result of the gas disk dispersal are debris disks. These disks are mainly made up of asteroid/comet/planet like bodies and dust but contain no or only very little amount of gas. This evolutionary phase can last for Gyr. However, it is still unclear if every gaseous disks ends up as a debris disk (see Matthews et al. 2014 for a review).

During these evolutionary stages also the dust component of the disk evolves. Recent discoveries like the horseshoe-shaped structures of larger dust particles in transition disks (e.g. van der Marel et al. 2013; Pérez et al. 2014a) or the spectacular dark rings in the thermal dust emission of HL Tau (ALMA Partnership et al. 2015; Pinte et al. 2016) and TW Hya (Andrews et al. 2016) clearly show this. The dust component also influences the gas disk. Dust is a strong opacity source in the optical and ultraviolet and therefore absorbs most of the stellar radiation. This significantly affects the disk thermal structure and chemistry (see Sects. 5 and 6). However, our focus here is on the gas disk and we refer the reader to Chap. 3 of this book and to the review by Testi et al. (2014) for more details on dust evolution.

We now briefly summarize the essential steps of disk evolution from formation to dispersal due to various erosion processes. Three major processes: accretion, winds/outflows/jets and planet formation contribute to the slow removal of disk material, determining the typical lifetime of disks.

The first and best studied process is the continuous accretion of gas and dust through the disk onto the star. There are several possible mechanisms which can drive matter flows in the disk, of which the most prominent ones are: the magneto-rotational instability (MRI), magnetically launched jet winds, magneto-centrifugally driven winds, gravitational instability and hydrodynamical instabilities (see Turner et al. 2014 for a review). The first mechanism has been the most promising one to explain increased disk viscosity required for the observed accretion rate. However, MRI requires a disk ionization fraction of $\approx 10^{-12}$ which is probably not reached everywhere in the disk (e.g. Cleeves et al. 2015). The second mechanism leads to fast jets and associated molecular outflows, but here the acceleration mechanism of disk material along magnetic fields is poorly understood, and it is also still a matter of debate whether the launching mechanism of molecular outflows is directly associated with jet acceleration (see review of Frank et al. 2014). The other processes (turbulence, winds and instabilities) are sensitive to heating and cooling processes in the disk. Therefore high quality observational data in combination with detailed thermo-chemical models are needed to derive quantities such as the ionization fraction and the gas temperature (see Sects. 4, 5 and 6). In summary, the efficiency of all above processes remains unclear. We also note that disk accretion might affect the chemical composition due to additional heating and transport of material from colder to warmer regions of the disk (see Sect. 6), and 
jet formation may lead to additional irradiation of disks from above (see Sect. 3.5). Generally, self-consistent thermo-chemical and (magneto-)hydrodynamic simulations including feedback are needed for a full understanding of these processes.

Especially in their earlier phases, most disks probably undergo more violent accretion phases. The so-called FU Orionis objects show strong luminosity outbursts (increase in luminosity by factors of 10 to 100 s). These outbursts typically last for decades or centuries. During this period the accretion rate rapidly increases from $\approx 10^{-7}$ to $\approx 10^{-4} \mathrm{M}_{\odot} \mathrm{yr}^{-1}$ (see Audard et al. 2014 for a review). The cause of this phenomenon is still unclear. Proposed explanations are disk instabilities (thermal, magneto-rotational and/or gravitational instabilities) and the perturbation of the disk by an external body. Another possibility is disk fragmentation and the infall of the formed fragments onto the star. These outbursts also have an impact on the chemical evolution of the disk (see Sects. 2 and 6). Although rare, repeated FU Ori-type accretion events may be crucial in star and disk evolution as they may bring a significant amount of matter onto the star, and may at the same time dominate disk mass-loss due to accretion on long timescales.

The second major mass-loss processes are via the formation of disk winds, either magnetically driven or irradiation driven. We distinguish between fast, probably magnetically driven jets with (at least in the Class-I phase) associated molecular outflows, and radiationdriven slow photoevaporative winds (see Sect. 8, Chap. 6 of this book and the review of Alexander et al. 2014). In the T Tauri phase, mass-loss rates in outflows/jets and photoevaporative winds amount to no more than about $10 \%$ of the stellar accretion rate (Hartigan et al. 1995; Cabrit 2002; Frank et al. 2014). However, in late evolutionary phases the photoevaporation rate may locally (at distances of several au from the star) exceed the disk accretion rate, which leads to the rapid formation of a gap and the subsequent erosion of the entire disk on time scales of only a few hundred kyr or $\approx 10 \%$ of the disk lifetime. This rapid-erosion model is compatible with the relatively rare occurrence of transitional disks with such gaps and eventually inner holes, compared to classical, optically thick disks. An example of gas photoevaporation are winds driven by X-ray or extreme-ultraviolet irradiation of the disk (see Sects. 5 and 8), leading to strong surface heating and ionization and consequently escape of ions. Photoevaporation might have an impact on the final appearance of a planetary systems. In fact the models of Ercolano and Rosotti (2015) suggest that the distribution of the semi-major axes of exoplanet orbits is sensitive to photo-evaporation as it affects planet-migration.

The third process eroding a disk is planet formation itself. The formation of gas giants requires an intact gas reservoir still to be present, which suggests that giants form to their full mass during the disk phase. The combined mass of the giant planets in the solar system amounts to about $0.13 \%$ of the solar mass, which provides a strict lower limit to the disk mass loss in the T Tauri phase of our Sun through planetary accretion. Giant planets may open gaps and form spiral-arm patterns, leading to a complex exchange of angular momentum between planet and disk, leading to planet migration. Meanwhile, solids also accumulate and grow up to Mars-sized planetary embryos, adding to the disk mass loss (for more details see the reviews by Baruteau et al. 2014 and Raymond et al. 2014). One spectacular observed example for this process might be the LkCa 15 transitional disks. Recent adaptive optics observations by Sallum et al. (2015) reveal up to three accreting giant proto-planets in the large inner cavity ( $r_{\text {cav }} \approx 50 \mathrm{au}$, Andrews et al. 2011) of this disk. At the same time the outer disk of this system still remains intact and shows similar observational signatures as classical disks (Öberg et al. 2011; Punzi et al. 2015).

As mentioned above, the relative importance of the disk erosion processes are unknown although all of them are observed, and all of them are expected to remove disk material in 
the range of maybe one tenth and about one percent of the solar mass during a typical disk lifetime (accretion and ejection rates of order $10^{-8} \mathrm{M}_{\odot} \mathrm{yr}^{-1}$ for a duration of $\sim 10^{6} \mathrm{yr}$ ).

Another important constraint for disk evolution and planet formation is the actual mass of the disk. Disk masses derived from millimeter continuum observations are in the range of $M_{\text {disk }} \approx 0.1 \mathrm{M}_{\odot}$ to $\lesssim 10^{-3} \mathrm{M}_{\odot}$ (Andrews and Williams 2005). However, disk masses derived from sub-millimeter fluxes can be underestimated by a factor of 2 , and in some cases up to a factor of 10 (Dunham et al. 2014).

Williams and Best (2014) proposed a method for a more direct measurement of the disk gas mass using $\mathrm{CO}$ isotopologue line ratios. Their results indicate rather low disk gas masses in the range of $10^{-2}$ to $10^{-3} \mathrm{M}_{\odot}$ and consequently, lower gas to dust mass ratios of the order of 10 , in contrast to the canonical value of 100 . However, direct disk gas mass measurements are difficult and are affected by complex chemical processes, which are not fully considered in Williams and Best (2014) (this is also true for CO, see Sect. 6 for more details). In contrast to the results of Williams and Best (2014), Bergin et al. (2013) reported a gas mass of $M_{\text {disk }} \gtrsim 5 \times 10^{-2} \mathrm{M}_{\odot}$ for the disk around TW Hya, using the HD molecule as the mass tracer. This result is especially interesting as TW Hya is supposed to be a rather old disk (3-10 Myr, Bergin et al. 2013). The derived disk mass would still allow to form a planetary system similar to our solar system.

To improve our knowledge of the gas disk structure and evolution, further observations are required. However, deriving disk properties directly from observations is not straightforward. Not all regions of the disk (e.g. the midplane) are directly observable and the chemical processing of disk material has to be considered for the interpretation of observations (see Sect. 6). However, the first results of the Atacama Large Millimeter Array (ALMA) have already significantly improved our knowledge of the gaseous disk (see Sect. 7), and most certainly will provide us with more interesting and surprising results in the near future.

From the above introduction of disk evolution and important physical processes it becomes clear that a review like this cannot cover this topic exhaustively. We rather focus on several important aspects and refer the interested reader to dedicated reviews for topics we do not cover in detail.

In Sect. 2 we start with a discussion about the early evolution of gravitationally unstable disks, their potential to form gas giants and their role for the episodic accretion scenario. Section 3 is about the radiative environment of the disk with a focus on stellar high-energy processes as they are in particular interesting for disk-ionization, heating and disk dispersal. In Sect. 4 we discuss in more detail disk ionization and the magneto-rotational instability as a potential driver of accretion. In Sect. 5 we briefly discuss the thermal structure of the disk, heating/cooling processes and disk photo-evaporation as a consequence of gas heating by high-energy radiation. The general chemical structure of classical $\mathrm{T}$ Tauri and Herbig $\mathrm{Ae} / \mathrm{Be}$ disks is described in Sect. 6. In this Section we also discuss in more detail X-ray chemistry, ice-lines and present a simple model for the chemical evolution during episodic accretion events. In Sect. 7 we give an overview of gas disk observations and in Sect. 8 we discuss observational constraints of photoevaporative winds. We conclude this chapter with a summary (Sect. 9).

\section{The Early Evolution of Circumstellar Disks}

Stars form from the gravitational collapse of dense molecular clouds, a larger fraction of which passes through a circumstellar disk owing to conservation of the net angular momentum of the collapsing cloud. Observational evidence and numerical simulations suggest that 
circumstellar disks can form as early as a few thousand years after the formation of the protostar (Vorobyov 2011b; Tobin et al. 2013) and sometimes even earlier than the protostar itself (Machida et al. 2010). In this early evolutionary phase, most of the mass reservoir is residing in the collapsing cloud, which lands onto the disk outer regions and drives the disk to the boundary of gravitational instability. Theoretical and numerical studies indicate that circumstellar disks can be prone to gravitational fragmentation in the outer regions if they form from cores of sufficient initial mass and angular momentum (Kratter et al. 2008; Vorobyov 2010; Stamatellos et al. 2011; Zhu et al. 2012). The infall of material from the collapsing core in the early stages of star formation triggers fragmentation in disks that otherwise might have been stable against fragmentation. The location of the fragmentation boundary (usually, at several tens of au from the central star) depends somewhat on the disk physics and is currently a subject of ongoing debate (Rafikov 2005; Boley 2009; Meru and Bate 2012).

In the subsequent protostellar phase of disk evolution, lasting until the parental cloud is accreted onto the star plus disk system or dissipated via star formation feedback, the complex interplay between mass infall onto the disk and disk transport processes, such as gravitational and viscous torques, leads to the formation of gravitationally unstable protostellar disks with radial and azimuthal profiles of density and temperature that are very different from those of the Minimum Mass Solar Nebula (Vorobyov 2011b; Bitsch et al. 2015). Nonaxisymmetric structures that emerge in the disk, such as spiral arms, clumps, and vortices, may serve as likely spots for dust accumulation and planetesimal formation, facilitating the formation of solid protoplanetary cores (Rice et al. 2004; Nayakshin 2011; Regály et al. 2012; Gibbons et al. 2015). Gravitational instability and fragmentation in the early evolution of protostellar disks brings about a wealth of phenomena, the most important of which are briefly reviewed below.

\subsection{The Burst Mode of Protostellar Accretion}

Numerical studies show that fragments forming in the outer regions of protostellar disks can be quickly driven into the inner regions and onto the star thanks to exchange of angular momentum with spiral arms and other fragments. This fast inward migration leads to luminosity outbursts as fragments fall onto the star and release their gravitational energy (Vorobyov and Basu 2006, 2010b; Machida et al. 2011). The magnitude, duration and frequency of the luminosity outbursts is similar to those typically observed in FU-Ori-type eruptions (Vorobyov and Basu 2015). Figure 1 illustrates the burst phenomenon showing the gas surface density distribution in a gravitationally unstable disk. ${ }^{1}$ In particular, each row of images presents a time sequence capturing individual luminosity bursts caused by fragments falling onto the star. The left, middle and right panels correspond to the time instances immediately preceding the burst, during the burst, and soon after the burst, respectively. The corresponding times are identified in each panel. The inserts in the left column zoom onto the fragments that are about to fall onto the star and the inserts in the right column present the time evolution of the total stellar luminosity during the shown time sequence. Strong luminosity bursts ranging from several tens to several hundreds of the solar luminosity are evident. Luminosity bursts in the model of disk gravitational instability and fragmentation are a robust phenomenon estimated to take place in at least $40 \%$ of protostellar disks (Vorobyov and Basu 2015).

\footnotetext{
${ }^{1}$ These data (and those provided in the subsequent sections) were obtained from numerical hydrodynamics simulations of collapsing pre-stellar cores using the method described in detail in Vorobyov and Basu (2015).
} 


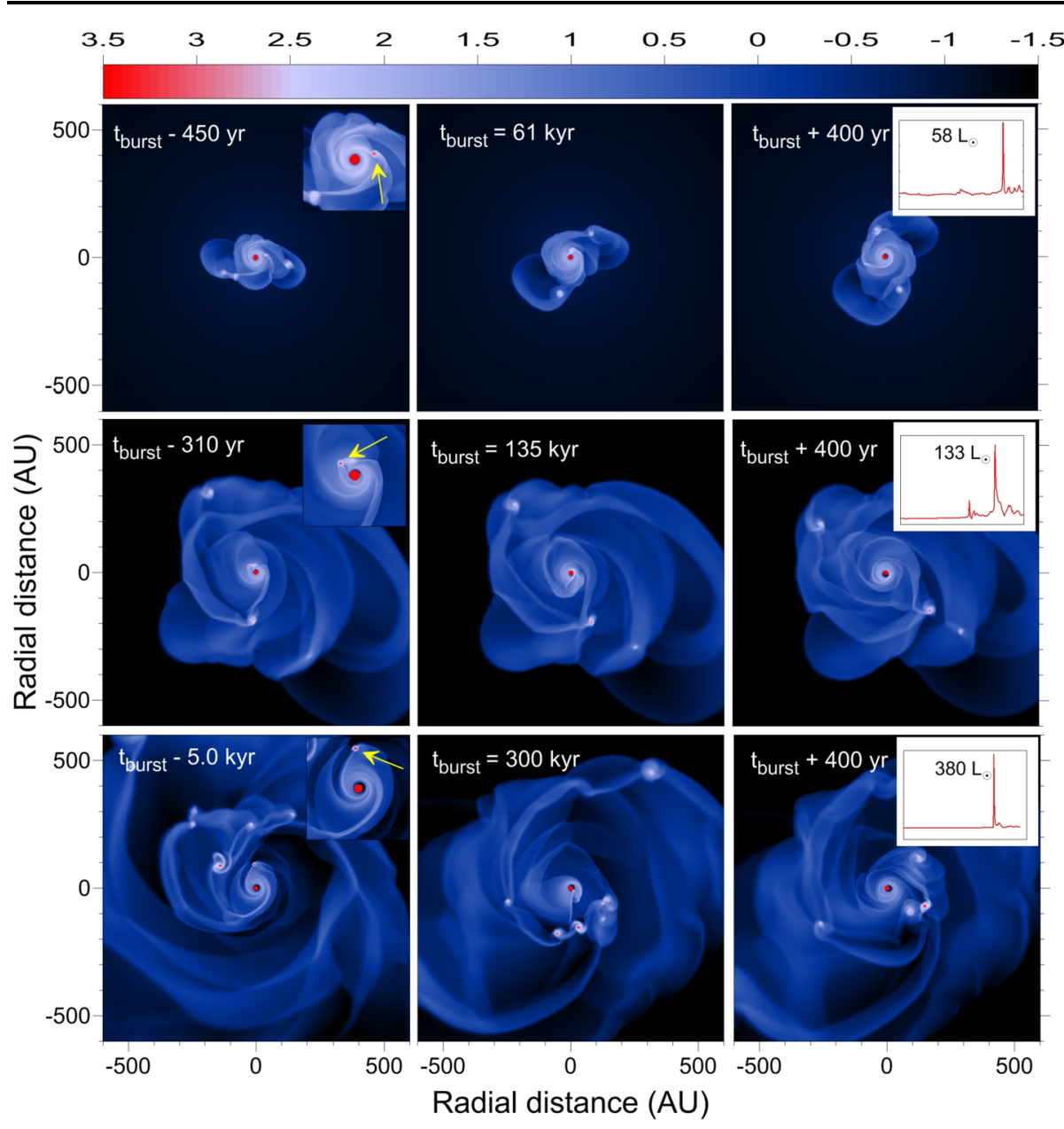

Fig. 1 Sequence of disk images capturing the inward migration of a clump onto the central star. The scale bar shows the gas surface density in $\mathrm{g} \mathrm{cm}^{-2}$. See the text for more detail

\subsection{Formation of Massive Gas Giants and Brown Dwarfs}

Another interesting phenomenon associated with disk gravitational fragmentation is the possibility for the formation of massive gas giant planet and brown dwarf companions to solar-type stars (Boss 1997, 2011; Stamatellos and Whitworth 2009; Boley et al. 2010; Nayakshin 2010; Kratter and Murray-Clay 2011; Rogers and Wadsley 2012). Numerical simulations capturing the long-term evolution of circumstellar disks (Vorobyov and Basu 2010a; Vorobyov 2013) show that under favorable conditions, i.e. in sufficiently massive disks that can experience fragmentation not only in the embedded but also $\mathrm{T}$ Tauri stages of star formation, some of the fragments may escape fast inward migration and mature into planetary or sub-stellar objects on wide orbits, similar to such systems as Fomalhaut or CT Chameleontis.

Figure 2 illustrates this process showing the evolution of a gravitationally unstable disk leading to fragmentation and ultimate survival of one of the fragments. The time elapsed 


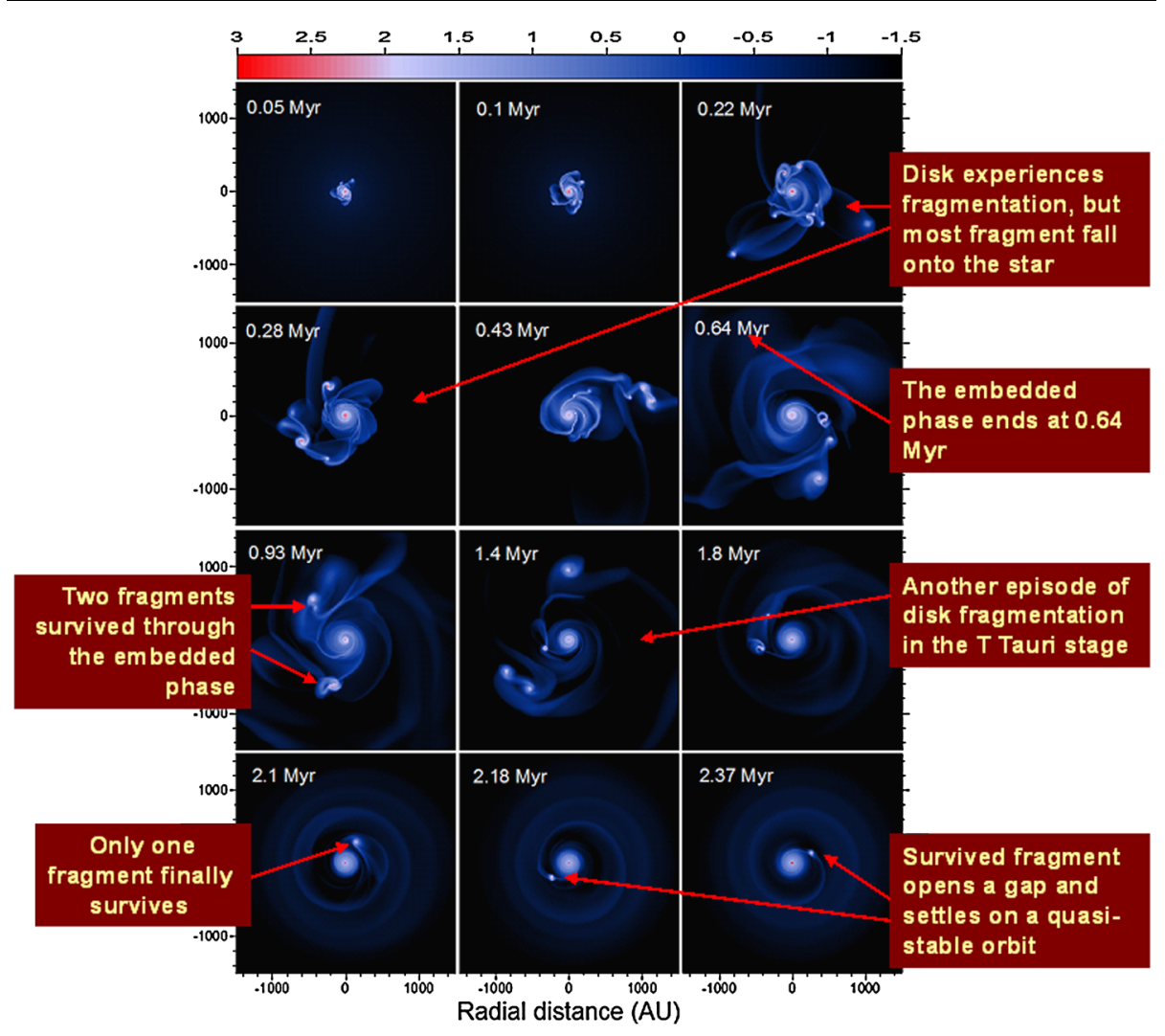

Fig. 2 Formation of a wide-orbit companion to a solar-mass star via disk fragmentation. The scale bar shows the gas surface density in $\mathrm{g} \mathrm{cm}^{-2}$. See text for more detail

since the formation of the central protostar is indicated in each panel. During the early phase $t \leq 0.65 \mathrm{Myr}$, when the forming star and disk are both embedded in the parental cloud, the disk experiences vigorous fragmentation, but most fragments are torqued onto the star producing strong luminosity bursts. After the end of the embedded phase, only two fragments are left in the disk. But a new (and last) episode of disk fragmentation takes place around $t=1.0 \mathrm{Myr}$ leading to the formation of two more fragments. However, only one fragment survives through the subsequent evolution and finally settles onto a wide-separation ( $\sim 300 \mathrm{au}$ ) orbit around the host star. The mass of the surviving fragment is $11 \mathrm{M}_{\mathrm{Jup}}$. In most cases, however, companions in the brown dwarf mass regime are formed. The described process operates in massive disks, but has a rather stochastic nature and does not always lead to the formation of a stable companion, even for otherwise similar initial conditions.

\subsection{Other Implications of Disk Fragmentation}

a) Ejection of fragments from the disk via many-body interaction. Close encounters between fragments in a protostellar disk can lead to the ejection of the least massive one into the intracluster medium. The ejected fragments, upon cooling and contraction, can form freely floating brown dwarfs and very-low-mass stars (Basu and Vorobyov 2012; Vorobyov 2016). This phenomenon is distinct from previously suggested scenarios of brown dwarf formation via 
disk fragmentation, wherein finished point-sized objects are ejected (Bate 2009; Stamatellos and Whitworth 2009), and is more consistent with observations of isolated proto-brown dwarf clumps (André et al. 2012; Palau et al. 2014).

b) Dust processing in the depths of massive fragments and formation of solid cores. Theoretical considerations and numerical hydrodynamics simulations of fragmenting disks (Boley 2009; Vorobyov 2011a; Nayakshin et al. 2011) demonstrate that gas temperatures in the depths of massive fragments may exceed $800 \mathrm{~K}$, thus initiating thermal annealing of amorphous silicates. If the tidal destruction timescale of these fragments is shorter than both the migration and dust sedimentation timescales, then the processed dust can be released at distances up to several hundred au, thus explaining the recent detection of crystalline silicates in the solar system comets. The fragments may also form solid cores in their interior before they are destroyed on their approach to the central star, providing a formation mechanism of protoplanetary cores that is alternative to the core accretion mechanism (Nayakshin 2010).

c) Solution of the luminosity problem. In the standard model of spherical core collapse (Shu 1977), the mass accretion rate is proportional to the cube of the sound speed. For typical gas temperatures in cloud cores on the order of 10-20 K, the corresponding accretion rate varies in the $(1-4) \times 10^{-6} \mathrm{M}_{\odot}$ range. A significant shortcoming of the standard model is the classic "luminosity problem", whereby accretion at the above rate produces accretion luminosities factors of 10-100 higher than typically observed for embedded protostars (Kenyon et al. 1990; Dunham et al. 2010). Accretion rates in gravitationally unstable disks can provide a solution to this problem. In the recent study, Dunham and Vorobyov (2012) calculated the radiative transfer of the collapsing cores throughout the full duration of the collapse using as inputs the disk, envelope, and stellar properties, as well as stellar mass accretion rates, predicted by the hydrodynamical simulations. They demonstrated that numerical hydrodynamics models that predict gradually declining accretion rates with episodic bursts caused by fragments migrating onto the star can account for the luminosity problem and explain a wide spread in luminosities of embedded sources.

d) Chemical tracers of recent luminosity bursts. As simple semi-analytic models show, luminosity outbursts similar in magnitude to those of FU-Orionis-type eruptions can raise the gas and dust temperature and drive significant chemical changes in the surrounding envelope and disk (Lee 2007; Visser and Bergin 2012; Kim et al. 2012; Visser et al. 2015). Numerical hydrodynamics simulations of disk-fragmentation-driven luminosity outbursts (Vorobyov et al. 2013) demonstrate that the CO ice can sublimate into the gas-phase in the inner envelope (a few $\times 10^{3}$ au) during the luminosity burst, remaining in the gas phase long after the burst has subsided. For the typical conditions in the inner envelope, the e-folding time for $\mathrm{CO}$ freeze-out onto dust grains is about $2500 \mathrm{yr}$. The fact that the $\mathrm{CO}$ freeze-out time can be much longer than the burst duration (100-200 yr) opens up a possibility for the observational detection of recent luminosity outbursts (see also Sect. 6).

\section{The Radiative Environment of Circumstellar Disks}

Radiation from the central star is a crucial driver of disk physics, in particular heating processes, ionization processes, and chemical reactions. The dust component in a disk plays a special role because it adds significant opacity to radiation. The balance between heating mechanisms (e.g., due to chemical reactions, photoionization processes) and cooling processes (line radiation from the gas and continuum radiation from the dust) determines the vertical temperature profile in the upper layers of the disk. Close to the midplane, gas 
and dust temperatures are equal due to coupling interactions between the two components ("accommodation", Chiang and Goldreich 1997). Both the chemical composition of the gas and the size distribution of dust grains are influencing the heating-cooling balance, so that complex radiation thermo-chemical simulations are required to model temperature profiles. Generally, the disk midplane is relatively cool. However, high-energy radiation leads to excessive ionization and heating of the disk surface layers in which gas and dust thermally decouple due to the low densities. These mechanisms are at the origin of gas-disk erosion and chemical processing of the gas components. We therefore concentrate on high-energy radiation and its effects on the gas disk in the following, while we refer the reader to the chapter on the dust disk for mechanisms involving the dust component (Chap. 3).

Circumstellar disks are immersed in various types of high-energy radiation and particle fluxes. Among them figure most prominently extreme-ultraviolet and X-ray radiation from hot stellar coronae; even some collimated jets have revealed prominent X-ray emission (Güdel et al. 2008); stellar flares are expected to add hard X-ray and gamma radiation, apart from an enhanced flux of energetic particles ("stellar cosmic rays"). High-energy radiation and particles act as ionization agents of disk gas and consequently heat disk gas to several thousand $\mathrm{K}$, thereby driving chemical reactions. Ionization and heating also act toward disk dispersal: the former by possibly inducing the magneto-rotational instability (MRI) through coupling of ionized disk gas to magnetic fields, and the latter by photoevaporating gas from the uppermost layers of the disk. The disk lifetime may thus well be controlled by highenergy processes taking place on or near the central star.

In the subsequent sections, we first briefly review the relevant radiation sources in stardisk systems, and then discuss ionization and heating and their role in gas disk structure and evolution.

\subsection{Stellar Coronal X-Rays}

X-ray and extreme-ultraviolet radiation from T Tauri stars (TTS) originates predominantly from a magnetized corona, as in the case of the Sun. The X-ray radiation level is much higher than the Sun's, saturating at a maximum level of about $10^{-3}$ times the stellar bolometric luminosity (Telleschi et al. 2007), i.e., at levels of $10^{29}-10^{31} \mathrm{erg} \mathrm{s}^{-1}$. Similar characteristics apply to Class I protostars. In the Orion region, the X-ray luminosities increase from protostars to TTS by about an order of magnitude, although the situation is unknown below 1-2 keV (Prisinzano et al. 2008).

\subsection{Stellar X-Ray Flares}

$\mathrm{X}$-ray flares are also common in TTS, producing plasma temperatures of up to $\approx 10^{8} \mathrm{~K}$ (Stelzer et al. 2007; Imanishi et al. 2001). Like in main-sequence stars, the flare peak temperature, $T_{\mathrm{p}}$, correlates with the peak emission measure, $E M_{\mathrm{p}}$ (or, by implication, the peak X-ray luminosity), roughly as (Güdel 2004)

$$
E M_{\mathrm{p}} \propto T_{\mathrm{p}}^{4.30 \pm 0.35}
$$

\subsection{X-Ray Fluorescence}

Photoionization of cool disk gas by X-rays above the Fe $\mathrm{K}$ edge at $7.11 \mathrm{keV}$ produces a prominent line feature at $6.4 \mathrm{keV}$ due to fluorescence. This feature has been detected in spectra of several classical TTS, in particular after intense X-ray flaring (Tsujimoto et al. 2005). But in at least one protostellar (Class I) case, strong fluorescence appears to be quasisteady rather than related to strong X-ray flares (Favata et al. 2005); perhaps, the $6.4 \mathrm{keV}$ line is excited by non-thermal electron impact in relatively dense, accreting magnetic loops. 


\subsection{Accretion-Induced X-Rays}

Accretion streams falling from the circumstellar disk toward the central star reach a maximum velocity corresponding to the free-fall velocity,

$$
v_{\mathrm{ff}}=\left(\frac{2 G M_{*}}{R}\right)^{1 / 2} \approx 620\left(\frac{M}{\mathrm{M}_{\odot}}\right)^{1 / 2}\left(\frac{R}{\mathrm{R}_{\odot}}\right)^{-1 / 2} \mathrm{~km} \mathrm{~s}^{-1} .
$$

This velocity is an upper limit as the material starts only at the inner border of the disk; a more realistic terminal speed is $v_{\mathrm{m}} \approx 0.8 v_{\mathrm{ff}}$ (Calvet and Gullbring 1998). On the stellar surface, a shock develops with a temperature of

$$
T_{\mathrm{s}}=\frac{3}{16 k} m_{\mathrm{p}} \mu v_{\mathrm{m}}^{2} \approx 3.5 \times 10^{6} \frac{M}{\mathrm{M}_{\odot}}\left(\frac{R}{\mathrm{R}_{\odot}}\right)^{-1} \mathrm{~K}
$$

( $m_{\mathrm{p}}$ is the proton mass and $\mu$ is the mean molecular weight, i.e., $\mu \approx 0.62$ for ionized gas). For typical T Tauri stars, $M=(0.1-1) \mathrm{M}_{\odot}, R=(0.5-2) \mathrm{R}_{\odot}$, and $M / R \approx(0.1-1) \mathrm{M}_{\odot} / \mathrm{R}_{\odot}$ and therefore $T_{\mathrm{s}} \approx(0.4-4) \times 10^{6} \mathrm{~K}$. The ensuing X-rays may be absorbed in the shock gas itself, contributing to its heating, but suggestive evidence for accretion-related X-ray production is available in the very soft X-ray range (e.g., based on flux excess in the O VII and Ne IX line triplets or indications of very high densities; Güdel and Telleschi 2007, Kastner et al. 2002).

\subsection{Jets and Herbig-Haro Objects}

Terminal shocks between jets and the interstellar medium ("Herbig-Haro (HH) objects"), are obvious candidates for X-ray production. Faint X-ray sources at the shock fronts of HH objects have indeed been found (e.g., Pravdo et al. 2001). However, these sources are very distant and probably of little relevance for the disk. On the other hand, in a few cases $\mathrm{X}$-ray sources have been detected in the launching jet regions no more than a few tens of au from the star; they are likely to form in shocks internal to the jet, and possibly also involve magnetic fields (e.g., Favata et al. 2002, Bally et al. 2003, Güdel et al. 2008). The faint low-resolution spectra are soft and indicate temperatures of a few million $\mathrm{K}$, compatible with shock velocities of a few $100 \mathrm{~km} \mathrm{~s}^{-1}$. These sources may be important as their X-ray luminosities compete with the stellar X-ray luminosity, but they are located vertically above the disk, providing their X-ray radiation relatively unobstructed access to the disk surfaces (in contrast to the grazing-incidence X-rays from the star), where they can drive heating, ionization and therefore photoevaporative flows or chemical reactions.

\subsection{Stellar High-Energy Particles}

Strong flares on the central star produce high fluxes of accelerated electrons and protons ("stellar cosmic rays") at planetary distances. From solar analogy and flare statistics, Feigelson et al. (2002) estimated a proton flux for the "T Tauri Sun" about $10^{5}$ times higher than at present (i.e., $10^{7}$ protons $\mathrm{cm}^{-2} \mathrm{~s}^{-1}$ at $1 \mathrm{au}$ ). This flux is probably present almost continuously given the high flare rate. Protostellar jets may also be excellent accelerators of high-energy particles (Padovani et al. 2015). There is indeed some indirect evidence for high-energy particles ejected by young stars (Ceccarelli et al. 2014). 
Fig. 3 Ionization rates for monochromatic X-rays as a function of column density vertically into a disk for a gas with standard interstellar composition, at a radius of 1 au. The source X-ray luminosity has been normalized to $1 \mathrm{erg} \mathrm{s}^{-1}$. The shoulder at deep levels are due to Compton scattering (from Igea and Glassgold 1999, (C) AAS. Reproduced with permission)

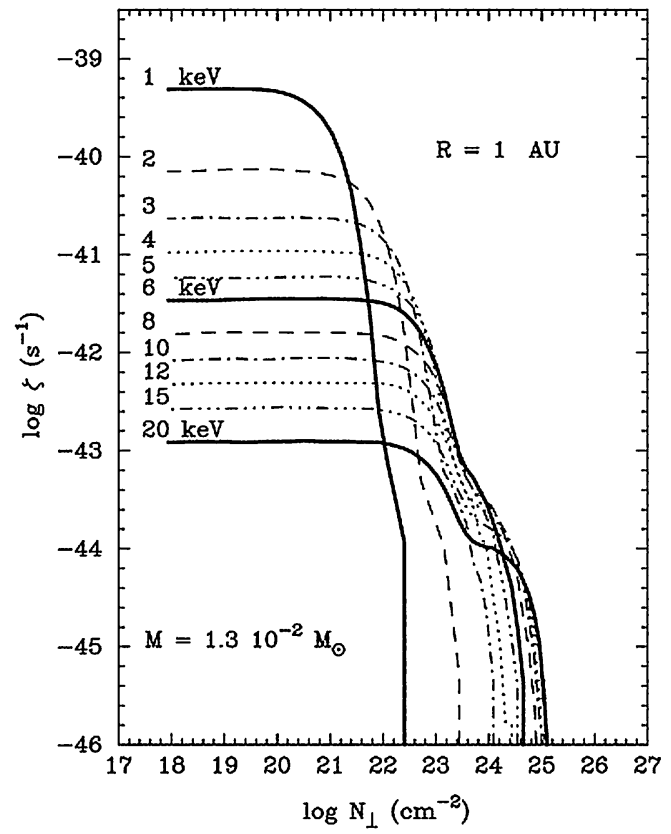

\section{Disk Ionization and the Magneto-Rotational Instability}

\subsection{The Ionization Structure of the Gas Disk}

The stellar X-ray spectrum is continuously being absorbed along the path from the star to the disk surface by the upper, tenuous layers of the disk gas and perhaps a disk wind. The dependence of the photon flux spectrum on photon energy $\varepsilon$ and position ( $r=$ radius of the disk segment) can be written as, for a geometrically thin, flat disk (Glassgold et al. 1997)

$$
F_{0}(\varepsilon, r)=\underbrace{\frac{R}{r} \frac{1}{2} \frac{1}{4 \pi r^{2}} \frac{L_{\mathrm{X}}}{k T}}_{f(r)} \underbrace{\frac{1}{\varepsilon} e^{-\varepsilon / k T}}_{g(\varepsilon)}, \quad \varepsilon>\varepsilon_{0}
$$

where the X-ray source is located at the center of the disk but vertically displaced by $R \ll r$; $R / r$ thus is the glancing angle at which the $\mathrm{X}$-rays strike the disk; the $1 / \varepsilon$ factor transforms energy flux to photon flux; the $1 /(k T)$ factor normalizes the total luminosity to $L_{\mathrm{X}}$, and $\varepsilon_{0}$ is introduced to simulate the low-energy cutoff due to absorption along the way from the star to the disk. When arriving at the disk surface, this flux is continuously attenuated according to the photoionization cross section. Essentially all of the initial X-ray energy $\varepsilon$ is used up to produce ionization, expending $\Delta \varepsilon \approx 37 \mathrm{eV}$ per ionization pair. Glassgold et al. (1997) and Igea and Glassgold (1999) performed numerical calculations of disk ionization for realistic disk profiles and shapes, also considering Compton scattering of energetic X-rays inside the disk. They essentially find that (see Fig. 3)

- the ionization rate by the softest X-rays at the disk surface at optical depth $\ll 1$ is approximately constant, owing to the spectral cutoff $\varepsilon_{0}$;

- the ionization rate rapidly decreases toward larger column depths where harder photons of the spectrum ionize predominantly heavier elements; 


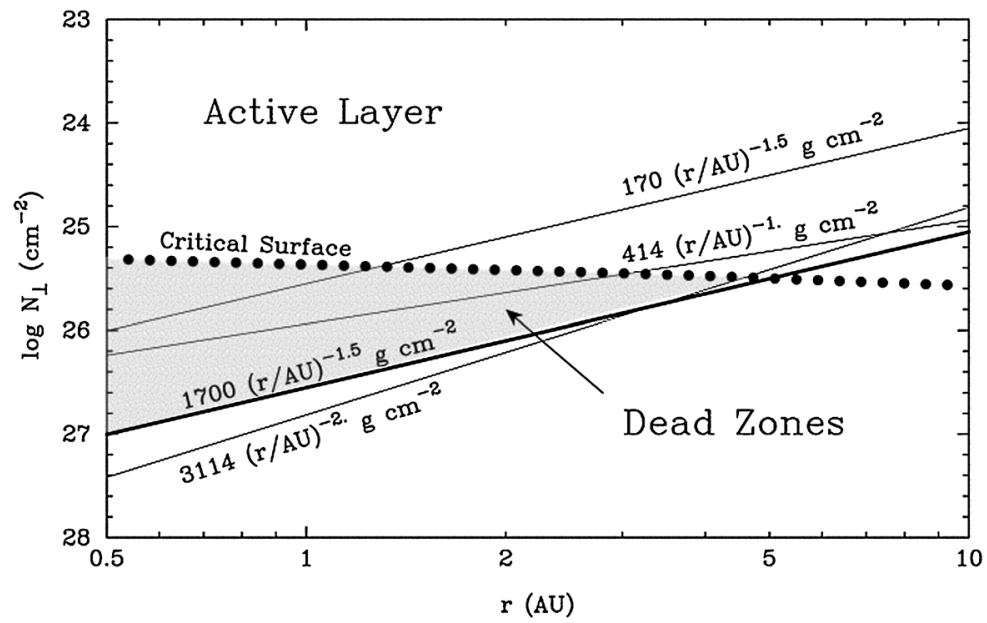

Fig. 4 Electron fraction in a disk due to X-ray ionization, shown in the radius $-N_{\perp}$ (vertical column density) plane. "Critical surface" marks the height below which the ionization fraction is too low to induce the magneto-rotational instability. The solid lines give disk mid-planes from different models (from Igea and Glassgold 1999; (C AAS. Reproduced with permission)

- harder spectra penetrate deeper into the disk and therefore ionize deeper layers;

- for a typical stellar coronal temperature of $k T=1 \mathrm{keV}$, the ionization degree corresponding to the adopted cosmic-ray ionization degree of $2 \times 10^{-17} \mathrm{~s}^{-1}$ is found at depths of $N_{\mathrm{H}} \approx 10^{23-25} \mathrm{~cm}^{-2}$ at disk radii of $\approx 10-1$ au.

- At column densities greater than $N_{\mathrm{H}} \approx 1.5 \times 10^{24} \mathrm{~cm}^{-2}$, the ionizing X-rays have energies $>10 \mathrm{keV}$ where the Compton cross section becomes larger than the absorption cross section, and the ionization rate begins to be altered by Compton scattering.

Calculations of the resulting ionization fraction $n$ (e) by Glassgold et al. (1997) and Igea and Glassgold (1999) (accounting for sedimentation of condensible heavy elements) show that the electron density $n$ (e) decreases rapidly toward the disk midplane; for a given vertical column density $N_{\perp}, n(\mathrm{e})$ also decreases with distance $r$; together, the disk ionization structure defines a wedge-shaped region around the inner disk mid-plane with its apex at the mid-plane at some outer radius, within which the ionization degree is very low ("dead zone"; Fig. 4).

\subsection{The Magneto-Rotational Instability}

Ionization of disk gas couples the disk gas to magnetic fields; this configuration in a differentially rotating disk is subject to the magneto-rotational instability (MRI; Balbus and Hawley 1991). The instability requires a minimum ionization degree; wherever this criterion is fulfilled, the disk is "active", other regions are called "dead zones" (Gammie 1996; see Fig. 4). MRI is held responsible for disk accretion. The criterion is given by the magnetic Reynolds number, $R e_{\mathrm{M}}$, expressing the relative importance between advection and magnetic diffusion,

$$
R e_{\mathrm{M}}=\frac{L v}{\eta} \approx 7.4 \times 10^{13} x_{\mathrm{e}} \alpha^{1 / 2}\left(\frac{R}{1 \mathrm{au}}\right)^{3 / 2}\left(\frac{T}{500 \mathrm{~K}}\right)\left(\frac{M}{\mathrm{M}_{\odot}}\right)^{-1 / 2}
$$


where $L$ is the characteristic length of variations in $B, \eta$ is the magnetic diffusivity, $v$ is the plasma flow velocity, and the last numerical expression is due to Gammie (1996), with $x_{\mathrm{e}}$ being the electron fraction and $\alpha$ the Shakura-Sunyaev viscosity parameter $\left(\alpha^{1 / 2}\right.$ is of order unity). For MRI to be efficient, the critical $R e_{\mathrm{M}}$ is about 100 , and this corresponds to an ionization fraction of $\approx 10^{-12}$ (see discussion in Fromang et al. 2002).

A significant part of the disk surface will couple sufficiently to magnetic fields to drive the instability, while the central layer around the midplane of the inner (few au) disk is insufficiently ionized and is therefore a "dead zone" (Fig. 4; Glassgold et al. 1997 and Igea and Glassgold 1999). However, considering also traces of metallic atoms, Fromang et al. (2002) find for a standard molecular $\alpha$ disk and $R e_{\mathrm{M} \text {, crit }}=100$ a dead zone extending from a fraction of an au out to 10-100 au. On the other hand, a small admixture of heavy-metal atoms is rapidly ionized by charge exchange with the molecular ions, while atomic ions recombine only slowly by radiation. For standard disks, an atomic abundance of heavy metals of as little as $10^{-6}-10^{-7}$ of the cosmic value may make the entire disk active, without any dead zone. Newer calculations including complex chemical networks, however, show the presence of a dead zone even if metals are present (Walsh et al. 2012; Cleeves et al. 2013a).

Cosmic rays are also efficient in ionizing disk material. Their importance for driving MRI was recognized in particular by Gammie (1996) who developed a model of layered accretion in $\mathrm{T}$ Tauri disks. The crucial question is that of the ionization rate. In the interstellar space, $\zeta_{\mathrm{CR}} \approx 10^{-17} \mathrm{~s}^{-1}$ (Spitzer and Tomasko 1968). The propagation of cosmic rays onto the disk surface is, however, a very significant problem. In the present solar system, low-energy cosmic rays are suppressed because of diffusion, convection and adiabatic cooling in the solar wind. The low-energy cosmic-ray spectrum is therefore highly uncertain for energies $\lesssim 10^{9} \mathrm{eV}$ due to modulation of the interstellar CR spectrum by the magnetized solar wind (filling the "heliosphere"). Various extrapolations down to lower energies provide largely diverging spectra (see summary by Cleeves et al. 2013a). The interstellar ionization rate is therefore unlikely to be appropriate for a protoplanetary disk environment, especially because $\mathrm{T}$ Tauri stars are, like the Sun, producing magnetic fields and are very likely to produce ionized winds. Cleeves et al. (2013a) estimate an attenuation of the cosmic ray flux at, say, 1 au from the star by $\approx 3$ orders of magnitude compared to the Sun and at least 6 orders of magnitude compared to interstellar conditions, resulting in a CR ionization rate of about $\zeta_{\mathrm{CR}} \approx 10^{-22}-10^{-20} \mathrm{~s}^{-1}$. This may result in much larger "dead zones" in protoplanetary disks, possibly reaching out to distances of $25 \mathrm{au}$.

\section{Heating of Disk Gas and Photoevaporation}

\subsection{Thermal Structure of a Gas Disk}

As laid out in Sect. 3, high-energy radiation strongly modifies the disk surface layers due to their strong heating rates and the decoupling of gas and dust in these low-density regions. In lower regions, dust and chemical processes are pivotal for the temperature profile, but in the upper disk atmospheres most of the heating is due to irradiation by stellar (or jet) ultraviolet, extreme-ultraviolet and X-ray photons.

In Fig. 5 we show the main heating/cooling processes for an exemplary model of a disk around a $\mathrm{T}$ Tauri star. The main stellar and disk properties of the model are: stellar mass $M_{*}=0.7 \mathrm{M}_{\odot}$, photospheric luminosity $L_{*}=1 \mathrm{~L}_{\odot}$, FUV luminosity $L_{\mathrm{FUV}}=$ $2.3 \times 10^{31} \mathrm{erg} \mathrm{s}^{-1}$, X-ray luminosity $L_{\mathrm{X}}=10^{30} \mathrm{erg} \mathrm{s}^{-1}$ and the disk mass is $M_{\text {disk }}=0.03 \mathrm{M}_{\odot}$ (for more details on the model see Sect. 6 and Table 1). In the upper layers of the disk the 

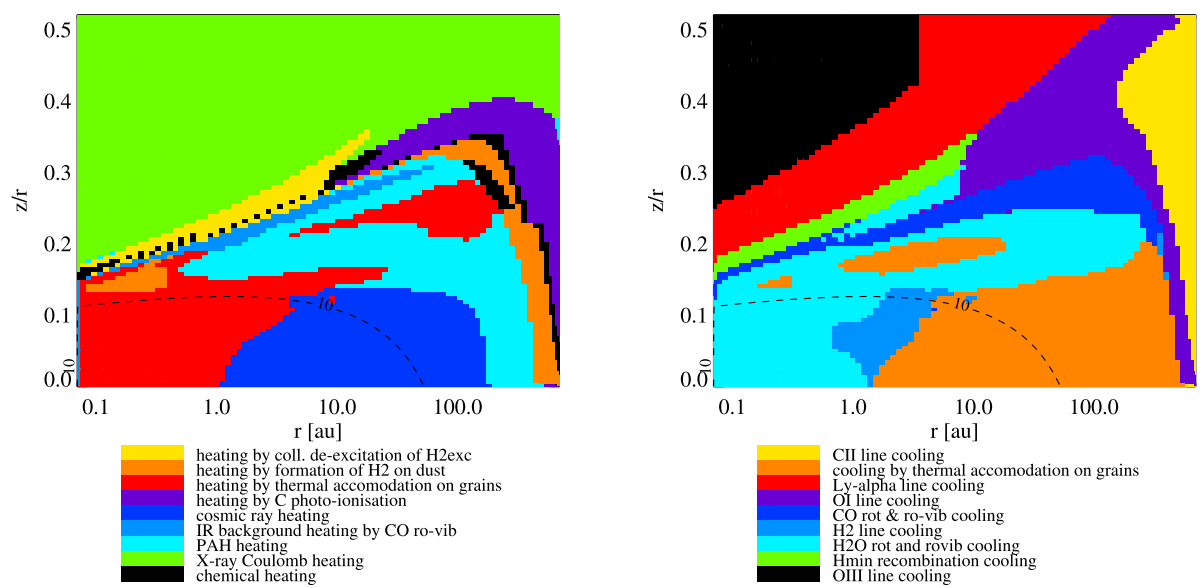

Fig. 5 Dominant heating (left panel) and cooling (right panel) processes for an exemplary ProDiMo model (Protoplanetary Disk Model, see Sect. 6 for details) of a disk around a T Tauri star. The plots show the dominant heating/cooling process at every point in the disk (identified by the different colors and the legend). The height of the disk is scaled by the radius $(z / r)$ to show also the details in the inner region. The dashed line indicates a visual extinction of 10

gas heating is dominated by radiation (e.g. X-ray Coulomb heating, C photo-ionisation due to FUV radiation). In the deeper layers the gas temperature is mainly controlled by the dust temperature due to collisions (heating/cooling by thermal accommodation on grains, Burke and Hollenbach 1983). The main cooling process in disks is line cooling. In the upper layers the cooling is dominated by emission of ionized or neutral atomic species like $\mathrm{O}$ or $\mathrm{C}^{+}$. In the transition region where $T_{\text {gas }}$ drops rapidly, at $N_{\perp} \approx(1-2) \times 10^{21} \mathrm{~cm}^{-2}$ (see Fig. 6), molecular line cooling is important. Prominent examples are $\mathrm{CO}$ and in deeper layers $\mathrm{H}_{2} \mathrm{O}$. $\mathrm{CO}$ is formed where $\mathrm{H}$ still abounds in its atomic form, and accelerates cooling through $\mathrm{CO}$ ro-vibrational and $\mathrm{CO}$ rotational emission, generating a strong temperature gradient; corresponding emission lines provide important observational diagnostics of disk heating and cooling.

We want to emphasize that the results shown in Fig. 5 are model dependent. For example the model of Glassgold et al. (2004) includes a viscous heating process which can be dominant in the transition region from the hot upper layers to the cool midplane (see Fig. 6). This process is not included in the model used for Fig. 5. However, all thermo-chemical models show the decoupling of the gas and dust temperature and the resulting high gas temperatures in the upper layers of the disk (e.g. Kamp and van Zadelhoff 2001; Gorti and Hollenbach 2004; Nomura and Millar 2005; Woitke et al. 2009). These results are qualitatively confirmed by Herschel observations (e.g. Bruderer et al. 2012; Tilling et al. 2012).

With the high spatial resolution of ALMA it now becomes possible to measure detailed radial temperature gradients in the disk. Schwarz et al. (2016) uses ALMA observations of several $\mathrm{CO}$ transitions (including isotopologues) to derive the radial temperature profile of the TW Hya disk. However, these observations mainly trace the warm molecular layer $(\approx 30-50 \mathrm{~K}$ see Sect. 6.1) and not necessarily the midplane of the disk. These kind of observations are required to improve and calibrate thermo-chemical disk models. However, it remains difficult to directly measure disk gas temperatures in particular in the deeper layers of the disk, and derived gas thermal structures of disks remain model dependent. 
Fig. 6 Gas and dust temperature profiles as a function of vertical column density into a disk, at 1 au from a central T Tauri star; apart from X-ray heating, viscous heating is present, characterized by the various $\alpha$ values ( $\alpha=0$ for no viscous heating; from Glassgold et al. 2004; (C) AAS. Reproduced with permission)

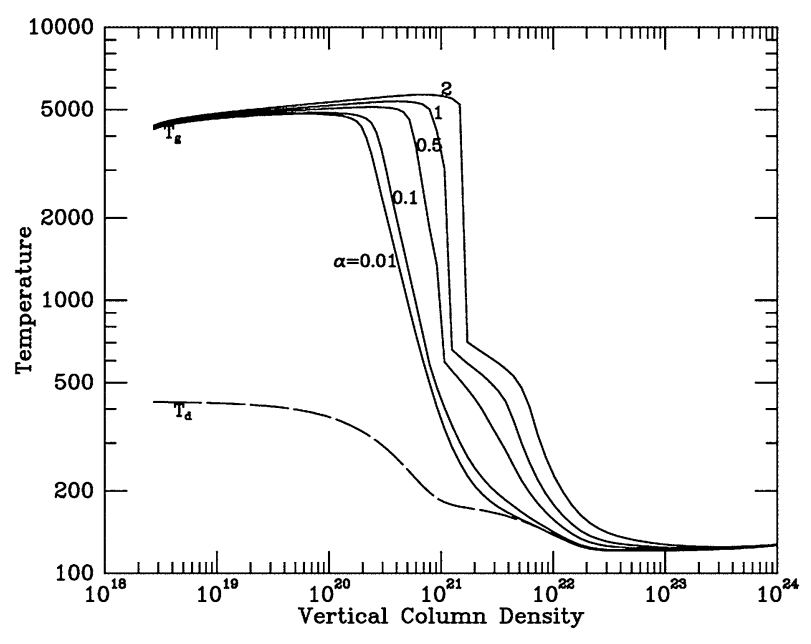

\subsection{X-Ray Heating}

$\mathrm{X}$-ray irradiation leads to substantial heating of the upper layers of protoplanetary disks. The heating energy per X-ray ionization event is about $\Delta \epsilon_{h} \approx 30 \mathrm{eV}$ (Glassgold et al. 2004). Realistic gas disk calculations irradiated by stellar X-rays show strong heating of the upper, atomic layers of the disk to several thousand $\mathrm{K}$ at 1 au (Fig. 6). This surface heating leads to a strong temperature inversion along the vertical direction through the disk, with a steep temperature decline at about $N_{\perp} \approx 10^{21} \mathrm{~cm}^{-2}$. Very close to the star, much hotter, fully ionized "disk coronae" at $T \approx 10^{6} \mathrm{~K}$ are formed (Ercolano et al. 2008). X-rays are very important heating agents out to $\sim 35-40$ au but disk ionization by stellar X-rays can be seen out to 190 au in such models.

\subsection{Disk Photoevaporation}

The heating of disk surface layers may produce temperatures at which individual particles escape from the stellar gravitational potential. This occurs once the mean particle energy, $\sim k T$, exceeds the gravitational binding energy of the particles with mass $m, G M_{*} m / R$. For a given temperature, this process of photoevaporation becomes effective beyond the gravitational radius, $R_{g}$, where the above criterion is fulfilled, i.e.,

$$
R_{g}=\frac{G M_{*}}{c_{s}^{2}}
$$

where $c_{s}=(k T / m)^{1 / 2}$ is the sound speed of the ionized gas.

Statistically, protoplanetary dust disks observed in the infrared disperse with a time scale of $\approx 3 \mathrm{Myr}$ (Mamajek 2009). However, the actual removal of an optically thick disk occurs rapidly at the end of this process, on a time scale of perhaps $0.5 \mathrm{Myr}$. This "two-time scale problem" was addressed by the "UV switch" model by Clarke et al. (2001), assuming that at some point in time, the disk accretion rate falls below the wind mass loss rate induced by photoevaporation at a certain radius of the disk. Subsequently, the resupply of the inner disk is suppressed and a gap is forming at this distance. The inner disk now disperses on its own, short viscous time scale. 
The EUV photoevaporation model of disks was first and extensively developed by Hollenbach et al. (1994) based on the diffuse recombination radiation field that ionizes the disk gas at large radii. The mass-loss rate depends on the flux of EUV photons, $\Phi$, and the mass of the central star, $M_{*}$, as

$$
\dot{M}_{w} \approx 4.4 \times 10^{-10}\left(\frac{\Phi}{10^{41} \mathrm{~s}^{-1}}\right)^{1 / 2}\left(\frac{M_{*}}{1 \mathrm{M}_{\odot}}\right)^{1 / 2} \mathrm{M}_{\odot} \mathrm{yr}^{-1} .
$$

If the escaping gas is flowing at sound speed $\left(c_{s} \approx 10 \mathrm{~km} \mathrm{~s}^{-1}\right)$, then the total mass loss rate at radius $R$ is

$$
\dot{\Sigma}_{w}(R)=2 \mu m_{\mathrm{H}} n_{0}(R) c_{s}(R)
$$

$\mu$ being the mean molecular weight of the gas, $\approx 2.3$ for a molecular gas, $m_{\mathrm{H}}$ the mass of a hydrogen atom, the factor of 2 accounting for the two sides of the disk, and $n_{0}$ being the number density of the disk gas.

Once the inner disk has drained, there is a significant contribution of direct radiation supporting the evaporation process in particular at the inner edge of the disk so that the outer disk also rapidly disperses. Alexander et al. (2006a) find a typical escape speed of $0.4 c_{s}$ and a mass-loss formula

$$
\begin{aligned}
\dot{M}_{w}\left(<R_{\text {out }}\right) \approx & 2.2 \times 10^{-9}\left(\frac{\Phi}{10^{41} \mathrm{~s}^{-1}}\right)^{1 / 2}\left(\frac{R_{\text {in }}}{3 \mathrm{au}}\right)^{1 / 2}\left(\frac{H / R}{0.05}\right)^{-1 / 2} \\
\times & {\left[1-\left(\frac{R_{\text {in }}}{R_{\text {out }}}\right)^{0.42}\right] \mathrm{M}_{\odot} \mathrm{yr}^{-1} }
\end{aligned}
$$

where $R_{\text {in }}$ and $R_{\text {out }}$ are the inner and outer disk radii, respectively, and $H / R$ is the ratio (assumed to be constant) between disk scale height $H$ and radius $R$. The effect of direct radiation thus enhances the mass loss rate by an order of magnitude above the estimate in Eq. (7). Numerical simulations confirm this behavior and suggest that at the end of the disk lifetime (several Myr), the disk rapidly disperses within $\gtrsim 10^{5} \mathrm{yr}$ (Alexander et al. 2006b).

EUV (Ly continuum) radiation is problematic because it may not reach the disk at all if disk winds absorb them early on. Also, Pascucci et al. (2014) claim that the EUV contribution is, based on observational evidence, too small to be effective for photoevaporation. Alternatively, soft X-rays may be relevant. Models based on Monte-Carlo methods for radiation show that $\mathrm{X}$-rays may indeed dominate the photoevaporation process, with wind mass-loss rates of order $10^{-8} \mathrm{M}_{\odot} \mathrm{yr}^{-1}$ (Ercolano et al. 2008). These studies were qualitatively and quantitatively supported by full hydrodynamic simulations by Owen et al. (2010) and Owen et al. (2011), emphasizing that the soft X-ray part of a stellar spectrum is the dominant driving mechanism for photoevaporation (rather than EUV alone). They find the mass-loss rate to scale linearly with $L_{\mathrm{X}}$. The important advantage of $\mathrm{X}$-rays here is their much larger penetration depths than for EUV, resulting in a much larger disk area subject to photoevaporation (out to $\sim 40 \mathrm{au}$ ) compared to the EUV case (for which the inner cutoff at $R_{g} \approx 5$ au for $10^{4} \mathrm{~K}$ gas limits the evaporation efficiency additionally). We will discuss observational evidence for photoevaporation of disks in Sect. 8.

\section{Chemical Processing of Disks}

Protoplanetary disks are considered to be the birthplaces of planets. It is likely that chemical processes occurring in those disks have an impact on the bodies formed in them. Molecular and atomic species are useful observational tracers of important physical properties of 


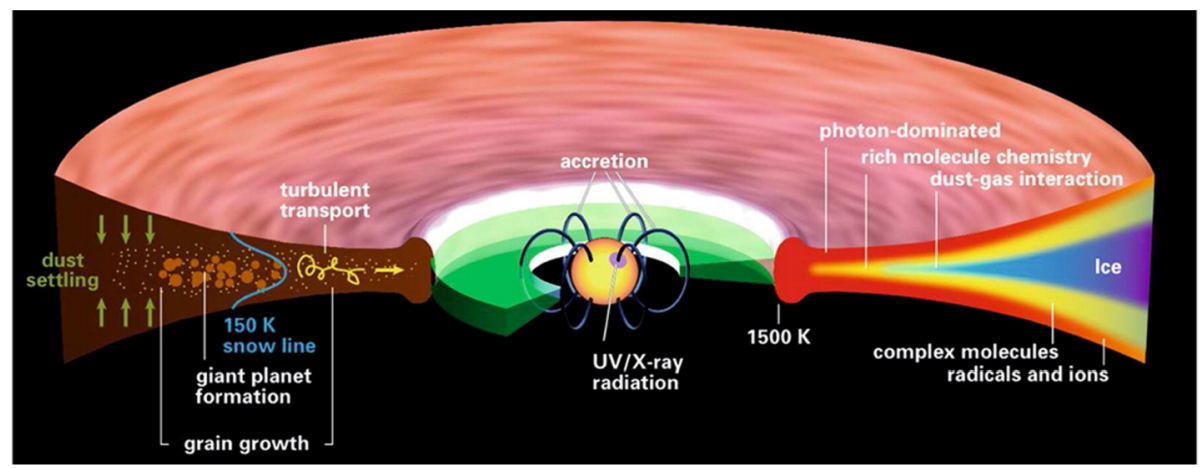

Fig. 7 Sketch of the physical and chemical structure of a protoplanetary disk around a sunlike star (age $\approx 1-5$ Myr). Adapted with permission from Henning and Semenov (2013), (C)(2013) American Chemical Society

disks like mass, temperature, disk structure and turbulence. These are important quantities for planet formation theories. For the interpretation of such observations a detailed understanding of chemical processes is required.

This is a challenging task as at least from a chemical point of view, disks are very complex structures. In gas disks temperatures can vary from $\approx 10$ to several $1000 \mathrm{~K}$ and densities from $\approx 10^{4}-10^{16} \mathrm{~cm}^{-3}$. Also external influences, in particular stellar radiation (e.g. UV, $\mathrm{X}$-rays, see Sect. 3) but also cosmic rays, alter the chemical composition of the disk. Furthermore, the dust component plays an important role (see Chap. 3 on the dust disk). Dust is an important opacity source shielding the deep regions of the disk from stellar radiation. In addition, dust allows for freeze-out of molecules (ice formation) in cold regions and the dust surface can act as a chemical catalyst for the formation of molecules (surface chemistry). Last but not least also dynamical processes like viscous evolution, turbulence, planet-disk interaction (e.g. via spiral arms) and the evolution of the star likely alter the chemical composition of the disk. Figure 7 gives an overview of the physical and chemical structure of a typical protoplanetary disk.

In terms of chemical disk modeling the inclusion of all relevant processes remains challenging. Most chemical disk models commonly assume a fixed (steady state) two dimensional disk structure and fixed stellar properties. So-called radiation thermo-chemical disk models also consistently solve for the gas temperature (heating/cooling processes) and often include detailed dust radiative transfer (e.g. Aikawa et al. 2002; Gorti and Hollenbach 2008; Woitke et al. 2009; Walsh et al. 2010; Bruderer et al. 2012; Du and Bergin 2014). Also the impact of turbulent mixing (e.g., Semenov et al. 2006; Furuya and Aikawa 2014) or dust evolution (e.g. Vasyunin et al. 2011; Akimkin et al. 2013) can be studied with such models. An extensive and complete review of this kind of models can be found in Henning and Semenov (2013) and Dutrey et al. (2014). Recently, chemical modeling has also been included in hydrodynamic simulations of disks. Examples are: Ilee et al. (2011) and Evans et al. (2015) who study the chemistry in the context of gravitationally unstable disks (e.g., in spiral arms) or Vorobyov et al. (2013) who study the impact of episodic accretion on the CO gas phase transition (see Sect. 2.3). Such models are computationally expensive and usually make simplifying assumptions about the radiative transfer and/or the chemistry. Nevertheless, such approaches are very powerful and give a first insight on the importance of dynamical processes on the chemical evolution of disks. 
Table 1 Main stellar and disk properties for the T Tauri and Herbig Ae/Be (where different) ProDiMo disk models

\begin{tabular}{lll}
\hline & T Tauri & Herbig Ae/Be \\
\hline Stellar mass & $0.7 \mathrm{M}_{\odot}$ & $2.1 \mathrm{M}_{\odot}$ \\
Stellar effective temperature & $4000 \mathrm{~K}$ & $6800 \mathrm{~K}$ \\
Stellar luminosity & $1.0 \mathrm{~L}_{\odot}$ & $32.0 \mathrm{~L}_{\odot}$ \\
FUV luminosity & $2.3 \times 10^{31} \mathrm{erg} \mathrm{s}^{-1}$ & $5.2 \times 10^{33} \mathrm{erg} \mathrm{s}^{-1}$ \\
X-ray luminosity & $10^{30} \mathrm{erg} \mathrm{s}^{-1}$ & $10^{29} \mathrm{erg} \mathrm{s}^{-1}$ \\
X-ray emission temperature & $2 \times 10^{7} \mathrm{~K}$ & \\
Disk gas mass & $0.03 \mathrm{M}_{\odot}$ & \\
Gas/dust mass ratio & 100 & $0.4 \mathrm{au}$ \\
Inner disk radius & $0.07 \mathrm{au}$ & \\
Characteristic radius & $100 \mathrm{au}$ & \\
Column density power index & 1.0 & \\
Scale height at $r=100$ au & $10 \mathrm{au}$ & \\
Flaring power index & 1.15 & \\
\hline
\end{tabular}

Here we do not discuss chemical models in detail but rather focus on the general chemical structure of the disk. For more complete reviews on disk chemical models and processes see Bergin et al. (2007), Henning and Semenov (2013), Dutrey et al. (2014). To qualitatively illustrate certain aspects discussed in this section, we use the results of two generic disk models, one T Tauri and one Herbig Ae/Be disk (see Table 1 for details). These two models should in particular illustrate the impact of the stellar properties on the chemical structure of the disk. Therefore we use for both models the same parameters for the disk (e.g. disk mass) with the exception of the inner disk radius. The inner disk radius is given by the dust condensation radius, and is therefore larger for the Herbig Ae/Be disk due to the stronger stellar radiation field.

We apply the radiation thermo-chemical disk code ProDiMo (Protoplanetary Disk Model, Woitke et al. 2009, Kamp et al. 2010, Thi et al. 2011, Aresu et al. 2011, Meijerink et al. 2012, Woitke et al. 2016), to calculate the thermal and chemical structure of the disk. We use time-dependent chemistry and evolve the chemistry up to 2 million years (chemical age of the disk). For the chemical reaction network we use the KIDA 2014 (Kinetic Database for Astrochemistry, Wakelam et al. 2015) database supplemented by additional chemical reactions included in ProDiMo. For the adsorption/desorption energies of the chemical species we use the data provided by the UMIST 2012 release (McElroy et al. 2013). More details on this particular model can be found in Woitke et al. (2016). We use the T Tauri and Herbig Ae/Be ProDiMo disk models to discuss the general chemical structure of disks (Sect. 6.1, Figs. 8 and 9) and the importance of ice lines for planet formation (Sect. 6.3, Fig. 10).

\subsection{Chemical Structure}

The following description of the chemical structure is strictly speaking only valid for T Tauri disks. However, the main chemical processes described are also valid for Herbig Ae/Be disks. We discuss the main differences between T Tauri and Herbig Ae/Be disks at the end of this section. 

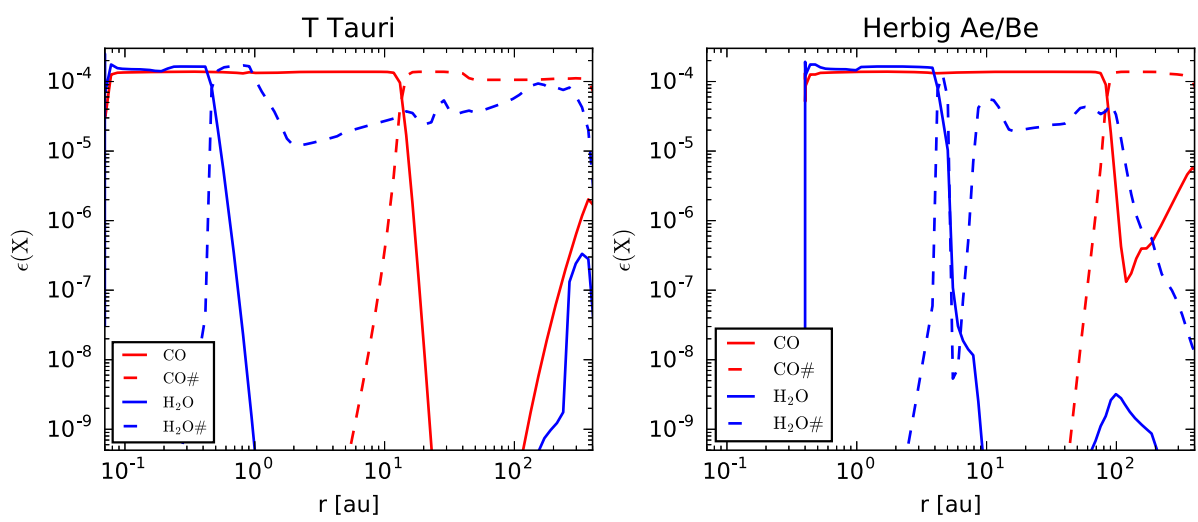

Fig. 8 Abundance of $\mathrm{CO}$ and water (relative to the total hydrogen number density) in gas and solid phase (as ice on the dust grains) in the midplane $(z=0 \mathrm{au})$ of the disk for the two generic ProDiMo disk models (left panel: T Tauri, right panel: Herbig Ae/Be, see also Table 1). The solid lines show the gas phase, the dashed lines the ice-phase abundances. The ice-line for water is at $\approx 0.5(5)$ au, the one for $\mathrm{CO}$ at $\approx 15(80)$ au for this particular T Tauri (Herbig Ae/Be) disk model. We note that the inner radius of the Herbig Ae/Be disk is at $0.4 \mathrm{au}$. For easier comparison we use the same scales for both plots

For the discussion of the chemical structure of the disk we divide the disk in different radial zones and vertical layers. From a chemical point of view the midplane of the disk contains three distinct zones:

- inner zone: extends up to a distance of a few au from the star; temperatures $T>100 \mathrm{~K}$; mostly shielded from radiation due to high optical depths;

- middle zone: extends from a few au up to $100 \mathrm{au} ; 100 \gtrsim T \gtrsim 20 \mathrm{~K}$; most species are adsorbed onto dust grains (e.g. water ice); shielded from radiation;

- outer zone: $T \lesssim 20 \mathrm{~K}$; most species freeze-out on dust grains; only partly shielded from radiation.

As an example we show in Fig. 8 the abundance of $\mathrm{CO}$ and water (for the gas and solid phase) in the midplane as a function of radius. The different locations of the ice-lines due to the different adsorption energies for $\mathrm{CO}$ and water are clearly visible. Also the re-increase of the gas phase abundance in the outer disk, in particular for $\mathrm{CO}$, is apparent.

In vertical direction it is common to define three distinct layers:

- midplane: see above; ice dominated chemistry (dust-gas interaction, surface chemistry);

- rich molecular layer: warm enough $(20 \lesssim T \lesssim 100 \mathrm{~K})$ so that most molecules are in gas phase; partly shielded from radiation; rich ion-neutral chemistry;

- photon-dominated layer: photochemistry (ionization, photo-dissociation); most species are in atomic form and ionized.

These three layers are also indicated in Fig. 7. In Fig. 9 we show, as an example, the two dimensional distribution of the $\mathrm{CO}$ molecule. The vertically layered molecular structure is clearly visible and is similar for other molecules.

Due to the strongly varying physical conditions the chemistry can be very different in the zones described above. For a proper treatment of the chemistry in the inner zone chemical reactions with high activation barriers can also become important (e.g. Harada et al. 2010), different from the usually colder interstellar conditions. Therefore, it can be expected that 

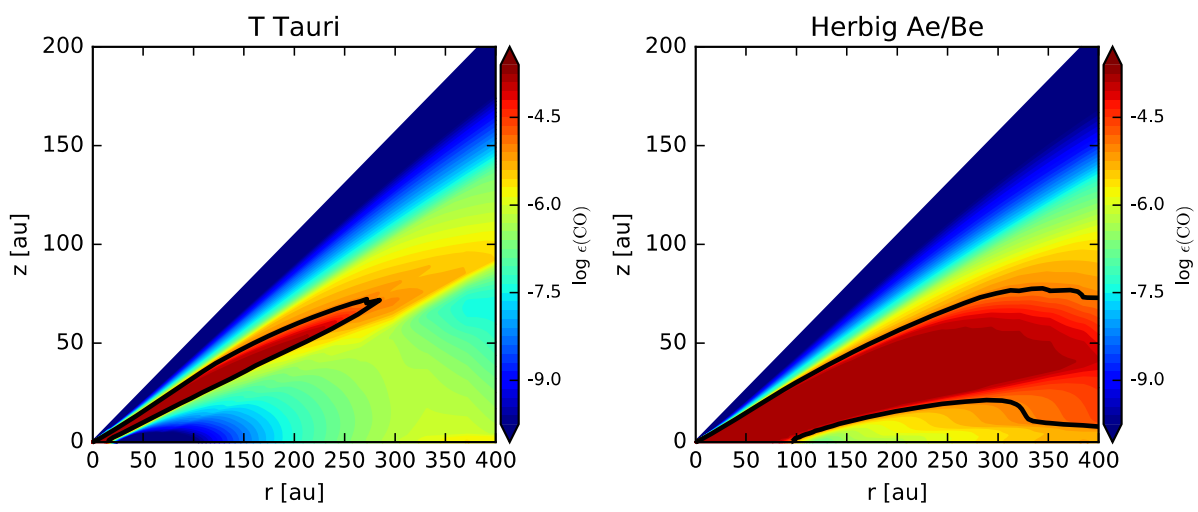

Fig. $9 \mathrm{CO}$ abundance $\epsilon(\mathrm{CO})$ (relative to the total hydrogen number density) for the two generic ProDiMo disk models (left panel: T Tauri, right panel: Herbig Ae/Be, see also Table 1). The black contour roughly encircles the warm molecular layer $\left(\epsilon(\mathrm{CO})=10^{-5}\right)$. Above this layer $\mathrm{CO}$ is photo-dissociated, below this layer CO freezes-out on dust grains. In the outer region of the midplane $(r \gtrsim 200 \mathrm{au}) \mathrm{CO}$ is also released back into the gas phase due to photo-desorption. The $\mathrm{CO}$ layer in the Herbig $\mathrm{Ae} / \mathrm{Be}$ disk is thicker and more extended compared to the T Tauri disk, as the disk is warmer due to the stronger stellar radiation field

the resulting chemical abundances are also different. Observations presented in Pontoppidan and Blevins (2014) show clear indications for this scenario. This work also shows that thermo-chemical models still have problems to explain the observed abundances. One reason might be that radial migration processes (e.g. large dust particles migrate inwards) are neglected. Migrating dust particles are probably coated in water ice. This ice is sublimates in the warmer conditions of the inner zone, enhancing the oxygen abundance which can subsequently also influence the chemistry. However, the thermo-chemical model of Walsh et al. (2014), for example, takes accretion flows into account, showing that also the abundance of complex molecules (e.g. methanol) can be strongly enhanced owing to the transport of ice-coated dust particles into the inner zone.

In the radiation shielded and much cooler middle zone of the midplane the important chemical drivers are cosmic rays and/or radionuclide ionization (and possibly also highenergy X-rays). The ionization rates of these processes are quite low, in the range of $\approx 10^{-20}-10^{-17} \mathrm{~s}^{-1}$ (e.g., Cleeves et al. 2013a,b) compared to the upper layers of the disk. Further photochemistry induced by the secondary UV field from cosmic rays (e.g. Gredel et al. 1989) becomes important in this region. A detailed study by Chaparro Molano and $\operatorname{Kamp}(2012 a, b)$ has shown that for disks proper dust and gas opacities have to be taken into account for the cosmic-ray induced photo processes. Their derived chemical abundances are compatible with measurements of the chemical composition of solar-system comets. The gas phase abundances of chemical species probably also influences the composition of gas giants that might be formed in this zone. One interesting quantity is the resulting $\mathrm{C} / \mathrm{O}$ gas phase abundance ratio which can be significantly altered, and therefore directly influences the chemical composition of giant planet atmospheres (e.g Helling et al. 2014).

Different from the middle zone, in the cold outer zone non-thermal desorption mechanisms (e.g. photo-desorption) may become important. Recent observations of $\mathrm{DCO}^{+}$in IM Lup suggest that non-thermal desorption (most likely photo-desorption due to stellar UV radiation) is efficient in the outer disk (Öberg et al. 2015; see also Sect. 7). In addition to the stellar UV radiation also the interstellar background field might play an important role for the $\mathrm{DCO}^{+}$abundance (Teague et al. 2015). Surface chemistry processes produce 
complex molecules on the dust surface (e.g. hydrogenation of $\mathrm{CO}$ to form $\mathrm{H}_{2} \mathrm{CO}$, Qi et al. 2013), and these molecules may subsequently be photo-desorbed. One prominent example for this suggested formation process is methanol (Walsh et al. 2010; Dutrey et al. 2014). Gas phase methanol was recently detected in the disk of TW Hya (Walsh et al. 2016). The observationally derived average abundances of methanol are up to two orders or magnitude lower compared to model predictions. This observations probably indicate a surface chemistry dominated formation pathway of methanol, but existing models seem to overestimate the efficiency of methanol formation and/or the efficiency of various desorption processes (Walsh et al. 2016).

Vertical turbulent mixing can transport ice coated dust particles to higher layers where photo-desorption is more efficient, and as a consequence gas phase abundances of complex molecules formed on dust grains are enhanced (Semenov and Wiebe 2011). On the other hand, vertical mixing can also decrease the abundance of complex organic molecules. Enhanced hydrogenation of radicals due to transport of atomic hydrogen to deeper layers can significantly suppress complex molecule formation (Furuya and Aikawa 2014).

In the warm molecular layer above the midplane one finds a very rich chemistry driven by higher temperatures, UV and X-ray radiation (Aikawa et al. 2002; Semenov et al. 2004; Henning and Semenov 2013). In our generic T Tauri disk model the layer is clearly visible in CO (Fig. 9). However, the location and the thickness of this layer probably vary from disk to disk and also depend on the dust properties. Vasyunin et al. (2011) have shown that in chemical models including large dust grains, this layer becomes thicker (e.g. extends deeper into the disk) compared to models considering ISM like dust properties (e.g. only small grains). Similar results are found by Akimkin et al. (2013) who used a self-consistent chemical model including dust evolution. They also find that on average, column densities of neutral molecules (e.g. $\mathrm{CO}, \mathrm{H}_{2} \mathrm{O}$ ) are enhanced in models considering dust evolution.

Another important chemical process in this layer is $\mathrm{CO}$ isotopologue chemistry (e.g. Visser et al. 2009), in particular isotopologue selective photo-dissociation. This chemical process may explain the anomalous ${ }^{17} \mathrm{O}$ and ${ }^{18} \mathrm{O}$ ratios measured in meteorites (Visser et al. 2009). CO isotope-selective photo-dissociation must also be considered if CO isotopologue line ratios are used to estimate disk gas masses. Thermo-chemical disk models of Miotello et al. (2014) show that using constant (throughout the disk) CO isotopologue ratios can lead to underestimating disk masses by up to an order of magnitude. Also, the possible depletion of gas phase CO in disks as suggested by Favre et al. (2013) and Bruderer et al. (2012) has to be taken into account. Although this depletion may be explained by isotope-selective photodissociation (in particular $\mathrm{C}^{18} \mathrm{O}$ ), chemical processes occurring closer to the midplane, like dissociation of $\mathrm{CO}$ by $\mathrm{He}^{+}$(Aikawa et al. 1998; Bergin et al. 2014; Furuya and Aikawa 2014) and/or surface chemistry (Reboussin et al. 2015) must also be considered if CO and its isotopologues are used as disk mass tracer.

Above the warm molecular layer direct ionization processes of atomic species and dissociation of molecules due to X-ray and UV photons are the main chemistry drivers. This layer shows typical properties of photo-dissociation regions (e.g. Hollenbach and Tielens 1999). A proper treatment of chemical processes in this region is important to determine the gas temperature, which is relevant for e.g. photo-evaporation in disks (see Sect. 5.3 and 8). Also X-rays play a major role in this layer ionizing noble gases like $\mathrm{Ne}$ and $\mathrm{Ar}$ and heating the gas to temperatures up to $10000 \mathrm{~K}$ (see Sect. 5).

T Tauri Versus Herbig Ae/Be Disk Chemistry A disk around a Herbig Ae/Be disk is on average warmer simply due to its stronger radiation field in the UV and optical wavelength regime. This has an impact on both the radial and vertical chemical structure of the disk. 
The locations of midplane ice lines are on average at larger radii in a Herbig Ae/Be disk than in a T Tauri disk (see Fig. 8). But also the vertical structure is affected. Compared to the $\mathrm{T}$ Tauri disk the warm molecular layer starts at slightly lower height (due to enhanced photo-dissociation) but extends much deeper towards the midplane of the disk. The warm molecular layer is mainly thicker due to the higher temperatures which prevents freeze-out of molecules (see Fig. 9).

Also molecular observations indicate that $\mathrm{T}$ Tauri disks have a larger cold chemistry reservoir compared to Herbig Ae/Be disks. The main indication here is the lower detection rates of cold chemistry tracers like $\mathrm{H}_{2} \mathrm{CO}$ (Öberg et al. 2011; Dutrey et al. 2014). Also the measured CO snowline location in HD 163296 of $\approx 90$ au (Qi et al. 2015) is a strong indication for warmer Herbig Ae/Be disks. As ice lines might play an important role for dust evolution (see Sect. 6.3), these chemical differences are most relevant for the study of early planet formation.

\subsection{X-Ray Driven Disk Ion Chemistry}

Given the importance of X-ray disk ionization, we briefly summarize chemical reactions directly induced by X-rays, in particular those illustrating some principal destruction routes of molecular ions. Among the principal ion destruction paths are the following, leading to "chemical heating" (Glassgold et al. 2012):

$$
\begin{aligned}
& \mathrm{H}_{2}^{+}+\mathrm{e} \rightarrow \mathrm{H}+\mathrm{H} \\
& \mathrm{H}_{2}^{+}+\mathrm{H}_{2} \rightarrow \mathrm{H}_{3}^{+}+\mathrm{H} \\
& \mathrm{H}_{3}^{+}+\mathrm{e} \rightarrow \mathrm{H}_{2}+\mathrm{H} \\
& \mathrm{H}_{3}^{+}+\mathrm{e} \rightarrow \mathrm{H}+\mathrm{H}+\mathrm{H} \\
& \mathrm{H}_{3}^{+}+\mathrm{CO} \rightarrow \mathrm{HCO}^{+}+\mathrm{H}_{2} \\
& \mathrm{H}_{3}^{+}+\mathrm{O} \rightarrow \mathrm{OH}^{+}+\mathrm{H}_{2} \\
& \mathrm{H}_{3}^{+}+\mathrm{H}_{2} \mathrm{O} \rightarrow \mathrm{H}_{3} \mathrm{O}^{+}+\mathrm{H} \\
& \mathrm{HCO}^{+}+\mathrm{e} \rightarrow \mathrm{CO}+\mathrm{H}
\end{aligned}
$$

$$
\begin{aligned}
& \mathrm{OH}^{+}+\mathrm{H}_{2} \rightarrow \mathrm{H}_{3} \mathrm{O}^{+} \\
& \mathrm{H}_{3} \mathrm{O}^{+}+\mathrm{e} \rightarrow \mathrm{H}_{2} \mathrm{O}+\mathrm{H} \\
& \mathrm{H}_{3} \mathrm{O}^{+}+\mathrm{e} \rightarrow \mathrm{OH}+\mathrm{H}_{2} \\
& \mathrm{H}_{3} \mathrm{O}^{+}+\mathrm{e} \rightarrow \mathrm{OH}+2 \mathrm{H} \\
& \mathrm{H}^{+}+\mathrm{H}_{2} \mathrm{O} \rightarrow \mathrm{H}_{2} \mathrm{O}^{+}+\mathrm{H} \\
& \mathrm{H}_{2} \mathrm{O}^{+}+\mathrm{H}_{2} \rightarrow \mathrm{H}_{3} \mathrm{O}^{+}+\mathrm{H} \\
& \mathrm{He}^{+}+\mathrm{CO} \rightarrow \mathrm{C}^{+}+\mathrm{O}+\mathrm{He} \\
& \mathrm{C}^{+}+\mathrm{H}_{2} \mathrm{O} \rightarrow \mathrm{HCO}^{+}+\mathrm{H}
\end{aligned}
$$

Complex thermo-chemical models of irradiated disks show that the abundances of neutral molecules such as $\mathrm{CO}, \mathrm{OH}$, and $\mathrm{H}_{2} \mathrm{O}$ are not overly sensitive to X-ray irradiation (a change by less than an order of magnitude over the range of many orders of magnitude in $L_{\mathrm{X}}$ ). In contrast, the X-ray sensitive ionization fraction in the upper layers of the disk strongly influences ion chemistry. The following reactions are important (Aresu et al. 2011; $M$ indicates the total mass of the species in the disk):

$$
\begin{aligned}
& \mathrm{H}_{2}+\mathrm{e} \rightarrow \mathrm{H}_{2}^{+} \\
& \mathrm{H}_{2}^{+}+\mathrm{H}_{2} \rightarrow \mathrm{H}_{3}^{+}+\mathrm{H} \\
& \mathrm{H}_{3}^{+}+\mathrm{O} \rightarrow \mathrm{OH}^{+}+\mathrm{H}_{2} \\
& \mathrm{H}_{3}^{+}+\mathrm{OH} \rightarrow \mathrm{H}_{2} \mathrm{O}^{+}+\mathrm{H}_{2} \\
& \mathrm{H}_{3}^{+}+\mathrm{H}_{2} \mathrm{O} \rightarrow \mathrm{H}_{3} \mathrm{O}^{+}+\mathrm{H}
\end{aligned}
$$

(secondary electrons from direct X-ray ionization)

$M(\mathrm{OH})$ grows approximately linearly with $L_{\mathrm{X}}$.

$M\left(\mathrm{H}_{2} \mathrm{O}^{+}\right)$grows somewhat less than $\mathrm{OH}^{+}$.

$M\left(\mathrm{H}_{3} \mathrm{O}^{+}\right)$grows by $10-100 \times$ for $1000 \times L_{\mathrm{X}}$.

Also $\mathrm{N}^{+}$reacts very sensitively to the presence of $\mathrm{X}$-rays and its total mass grows about linearly with $L_{\mathrm{X}}$. These reactions also spread in disk area, being pushed to larger distances as $L_{\mathrm{X}}$ grows. A special case of interest is the fine-structure emission of [O I]. The models clearly predict a linear growth of its line flux with the sum of $L_{\mathrm{X}}+L_{\mathrm{UV}}$. Whichever of the two luminosities dominates determines the correlation. Because of the usually appreciable 
UV field around T Tauri stars, an X-ray dependence comes into play only above $L_{\mathrm{X}} \approx$ $10^{30} \mathrm{erg} \mathrm{s}^{-1}$ (Aresu et al. 2014). Observations support the flux levels approximately although the correlation is not evident, possibly due to some influence of other parameters such as grain size distribution, surface density distribution, etc. in real objects.

\subsection{Ice Lines}

Ice lines (or condensation fronts) probably play a significant role in planet formation processes (e.g. through dust growth) but are also relevant for the final composition of bodies formed in the disk.

Recently Qi et al. (2013) reported the imaging of the CO snowline in the TW Hya disk via observations of the $\mathrm{N}_{2} \mathrm{H}^{+}$ion. $\mathrm{N}_{2} \mathrm{H}^{+}$is efficiently destroyed by proton transfer to $\mathrm{CO}$

$$
\mathrm{N}_{2} \mathrm{H}^{+}+\mathrm{CO} \rightarrow \mathrm{HCO}^{+}+\mathrm{N}_{2} \text {. }
$$

Therefore $\mathrm{N}_{2} \mathrm{H}^{+}$can only be abundant in regions with low $\mathrm{CO}$ gas phase abundances. The resulting ring like structure visible in images of the $\mathrm{N}_{2} \mathrm{H}^{+}$line emissions is also, at least qualitatively, reproduced by thermo-chemical disk models (e.g. Cleeves et al. 2015; Aikawa et al. 2015). The situation is illustrated in Fig. 10 for our generic T Tauri disk model. However, Aikawa et al. (2015) discuss other relevant chemical processes influencing the resulting $\mathrm{N}_{2} \mathrm{H}^{+}$distribution. One example is the so called sink effect, where $\mathrm{CO}$ is converted to less volatile species in regions with temperatures exceeding the sublimation temperature of $\mathrm{CO}$ (e.g. $>20 \mathrm{~K}$ ). Therefore, the peak of the $\mathrm{N}_{2} \mathrm{H}^{+}$abundance may be slightly below the $\mathrm{CO}$ sublimation radius.

There is a similar mechanism involving water, where $\mathrm{HCO}^{+}$is destroyed by water vapor. This mechanism was proposed to explain the ring-like appearance of the $\mathrm{H}^{13} \mathrm{CO}^{+}$emission in young protostars experiencing a recent luminosity outburst (Jørgensen et al. 2013). Such outbursts significantly enhance the gas phase water abundance, leading to subsequent destruction of $\mathrm{HCO}^{+}$. This chemical process is also relevant for disks; however, more detailed studies are required to investigate its efficiency under disk conditions. Furthermore, direct imaging of the water ice line via $\mathrm{HCO}^{+}$would require a much higher spatial resolution, as the water ice line is located much closer to the star (typically around 1 au, see also Fig. 8).
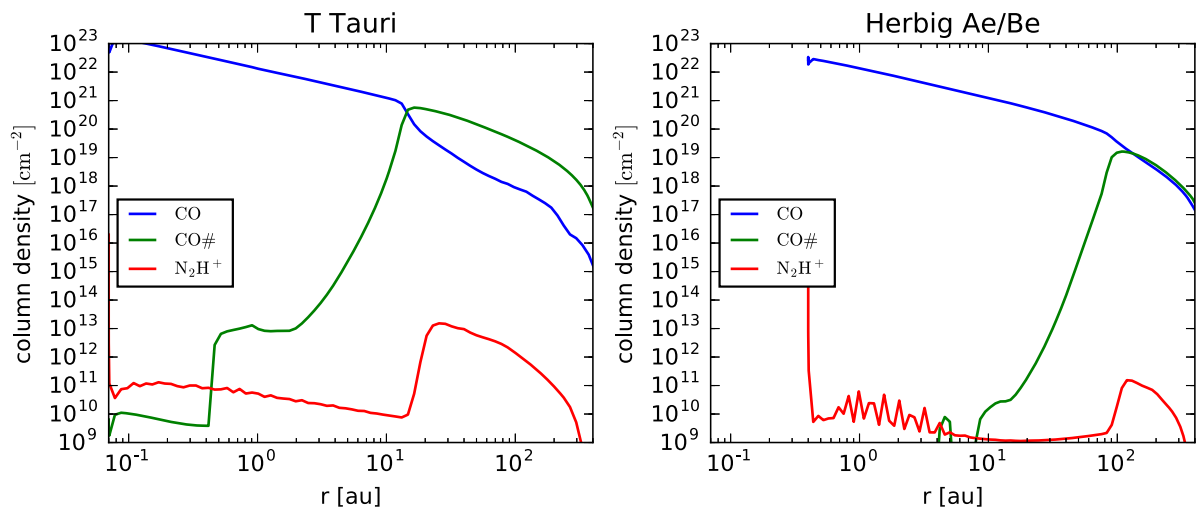

Fig. 10 Vertical column densities for $\mathrm{CO}, \mathrm{CO}$ ice $(\mathrm{CO \#})$ and $\mathrm{N}_{2} \mathrm{H}^{+}$as a function of radius for the two generic ProDiMo disk models (left panel: T Tauri, right panel: Herbig Ae/Be, see also Table 1). The jump in the $\mathrm{N}_{2} \mathrm{H}^{+}$column density at the CO ice lines (at $\approx 15$ and $80 \mathrm{au}$ ) is visible in both models but less pronounced in the Herbig Ae/Be disk 
The recent work of Zhang et al. (2015) suggests that the locations of the dark rings visible in ALMA continuum observations of HL Tau (ALMA Partnership et al. 2015) coincide with the expected ice line locations of several molecules. In particular, the locations of the $\mathrm{H}_{2} \mathrm{O}$, $\mathrm{NH}_{3}$ and $\mathrm{CO}$ ice lines agree well with the three most prominent dips in the radial surface brightness distribution. The same authors argue that around the location of the ice lines, enhanced pebble-growth (particles larger than a few $\mathrm{cm}$ ) quickly reduces the number of $\mathrm{mm}$-sized particles, which would explain the lack of emission inside the rings.

\subsection{FU Orions Like Outburst Scenario}

As an example for the impact of stellar/disk evolution on the chemical structure we discuss the impact of luminosity outbursts (see Sect. 2.1). During these outbursts the total luminosity of the system can increase by more than a factor of 100 compared to quiescent phases. The most prominent example for such outbursts is probably FU Orionis (e.g., Zhu et al. 2007). As already mentioned, Vorobyov et al. (2013) studied the impact of such outbursts on the gas phase transition of $\mathrm{CO}$ in a complex hydrodynamic model with a simplified chemistry (only thermal adsorption/desorption processes for $\mathrm{CO}$ and $\mathrm{CO}_{2}$ were considered).

We present here a simple disk+envelope model using the radiation thermo-chemical disk code ProDiMo. The modeling approach is similar to Visser et al. (2015), who studied the impact of luminosity outbursts on the chemical structure of envelopes around young protostars. Different from the model of Visser et al. (2015), our model considers an outburst scenario at a later evolutionary stage when a prominent Keplerian disk has already formed (e.g. similar to FU Orionis). In addition to the disk component, a remnant envelope structure is included (see Table 2). For the outburst we assume an instantaneous increase of the stellar luminosity by a factor of 100 lasting for 100 years.

Table 2 Main stellar and structure properties for the $\mathrm{FU}$ Orions like outburst model

\begin{tabular}{ll}
\hline Quantity & Value \\
\hline Stellar mass & $0.5 \mathrm{M}_{\odot}$ \\
Stellar effective temperature & $4000 \mathrm{~K}$ \\
Stellar luminosity & $1.0,100.0 \mathrm{~L}_{\odot}$ \\
FUV luminosity & $1.1 \times 10^{31} \mathrm{erg} \mathrm{s}^{-1}$ \\
X-ray luminosity & $10^{30} \mathrm{erg} \mathrm{s}^{-1}$ \\
X-ray emission temperature & $2 \times 10^{7} \mathrm{~K}$ \\
Disk gas mass & $0.02 \mathrm{M}_{\odot}$ \\
Gas/dust mass ratio & 100 \\
Inner disk radius & $0.6 \mathrm{au}$ \\
Outer disk radius & $200 \mathrm{au}$ \\
Column density power index & 1.0 \\
Scale height at $r=100$ au & $10 \mathrm{au}$ \\
Flaring power index & 1.15 \\
Envelope gas mass & $0.02 \mathrm{M} \odot$ \\
Inner envelope radius & $0.6 \mathrm{au}$ \\
Outer envelope radius & $3500 \mathrm{au}$ \\
Cavity opening angle & $30 \mathrm{deg}$ \\
\hline
\end{tabular}



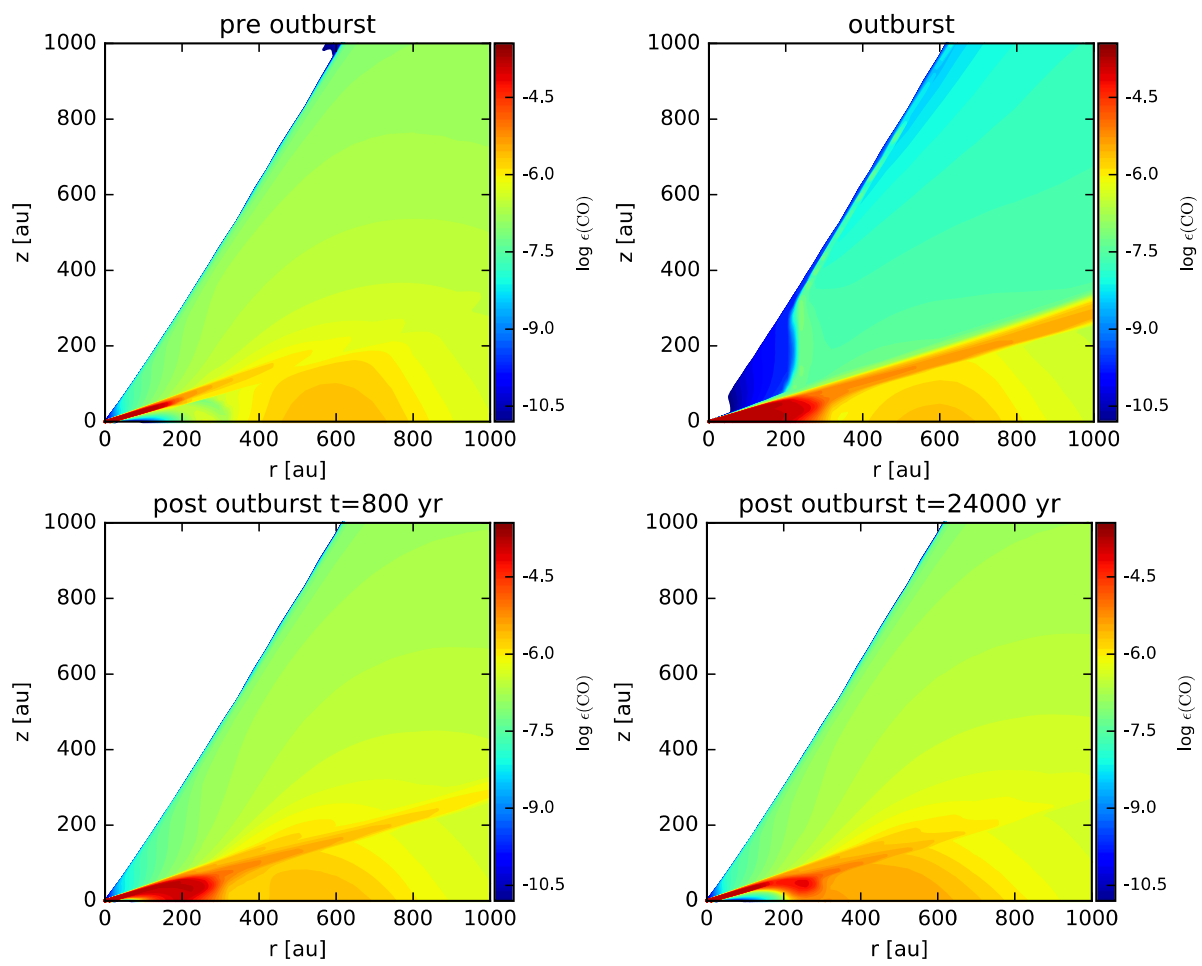

Fig. 11 Time evolution of the CO gas phase abundance $\epsilon(\mathrm{CO})$ for a FU Ori like outburst scenario. The structure includes a disk component and a remnant envelope (see Table 2 for details). The top left plot shows $\epsilon(\mathrm{CO})$ for the quiescent phase (stellar luminosity $L_{*}=1 \mathrm{~L}_{\odot}$ ), the top right plot during the outburst phase $\left(L_{*}=100 \mathrm{~L}_{\odot}\right)$, the bottom left and right plots show the abundance $800 \mathrm{yr}$ and $24000 \mathrm{yr}$ after the outburst, respectively

In Fig. 11 we show the $\mathrm{CO}$ gas phase abundance before the outburst, during the outburst, and $800 \mathrm{yr}$ and $24000 \mathrm{yr}$ after the outburst. Due to the increased luminosity the disk is heated and $\mathrm{CO}$ sublimates almost everywhere in the disk. In the upper layers of the disk+envelope structure $\mathrm{CO}$ is also dissociated by the enhanced stellar UV field. However, due to the warmer conditions the warm $\mathrm{CO}$ layer extends further out into the envelope structure (up to $r \approx 2000 \mathrm{au}$ ). Closer to the midplane the envelope structure ( $r>200 \mathrm{au}$ ) is shielded by the disk and the $\mathrm{CO}$ abundance does not change significantly during the outburst (top right plot in Fig. 11).

After the outburst $\mathrm{CO}$ freezes out again. In the inner disk this happens quite quickly, whereas in the outer disk $(r \approx 100 \mathrm{au})$ the freeze-out timescales are longer $(\approx 1000 \mathrm{yr})$ due to lower densities. In the envelope structure the gas phase $\mathrm{CO}$ abundance is still enhanced after $24000 \mathrm{yr}$ after the outburst. Approximately $100000 \mathrm{yr}$ after the outburst even in the outer envelope the gas phase $\mathrm{CO}$ abundance reaches again its pre-outburst level.

This is a very simplified approach for modeling an outburst scenario as it ignores the possibly significant impact on the disk structure during these violent phases. However, such models allow us to study the chemical evolution in more detail (e.g. impact on other species than $\mathrm{CO}$ ). The treatment of detailed dust radiative transfer also allows us to study the impact of different dust properties (size distribution, opacities) that may, for example, change the freeze-out timescales. Although such models are not very realistic in terms of the dynamical 
evolution, they can identify important chemical processes that can subsequently be incorporated in more complex hydrodynamical models. Furthermore, such models can be used to study the impact on observables in particular molecular line emission.

\section{Observations of the Gas Content of Disks}

Gas represents the bulk of the mass content in disks. However, from an observational point of view the gas (in particular the gas mass) is difficult to trace. Most molecular gas lines originate within a thin layer, limited by the adsorption of molecules onto dust grains towards the disk midplane, and the influence of high energy radiation in the upper disk layers that dissociates molecules and ionizes atoms (see Sect. 4 and 6).

In the radial direction, the gas content in disks extends closer to the protostar than the dust component and the outer radius is influenced by the disk geometry and the radiation from the central source, although very recent observations suggest that desorption may occur in the outer disk due to non-thermal processes (Öberg et al. 2015). Still, radiation from the protostar is the main source of energy defining the gas excitation, especially in the inner disk, but also as the main catalyst driving diverse chemical pathways. In this respect, higher excitation conditions corresponding to higher kinetic temperatures should occur closer to the protostar, while densities of different species shall probe the vertical structure of disk. In this simplified view the most common molecules detected in disks are represented in Fig. 12, as a function of radius and height in a disk. For more details on gas line diagnostics see also the reviews of Najita et al. (2007) and Carmona (2010) for the inner disk and Dutrey et al. (2014) for the outer disk.

The composition of the gas content in disks is subject to change, and chemical reactions play naturally a pivotal role, along with phase transitions (adsorption/desorption onto dust grains). It is therefore that chemistry in disks can trace different physical conditions but also plays an important role in controlling the disk dynamics. Consequently, understanding the chemical evolution of disks is central to decode the conditions for planet formation and habitability. The study of chemistry in disks in combination with the detailed study of the chemical compositions of solar system bodies is fundamental in understanding the conditions for life on Earth.

Fig. 12 Commonly observed gas lines, represented as a function of the excitation conditions (temperature, density), corresponding roughly to the radial and vertical structure in a $T$ Tauri disk. We note that the temperature scale does not refer to the disk midplane, but is just indicative of the (excitation) temperature as a function of distance from the star (from Dionatos 2015)

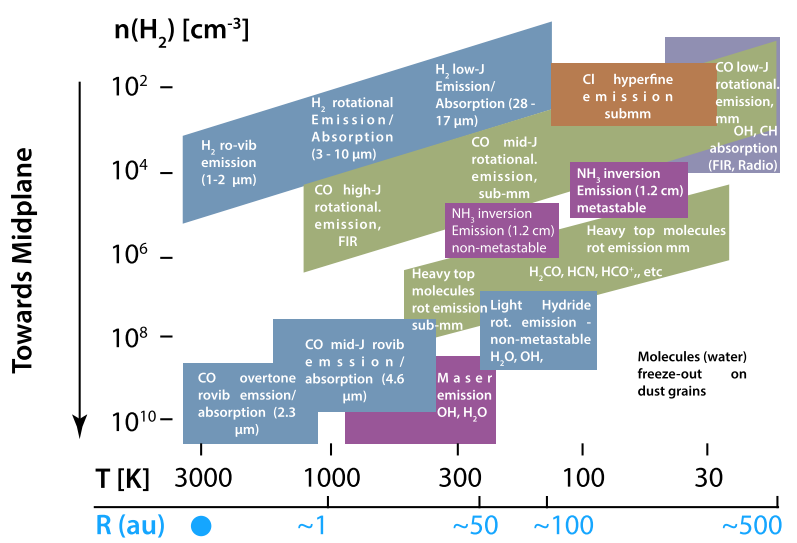



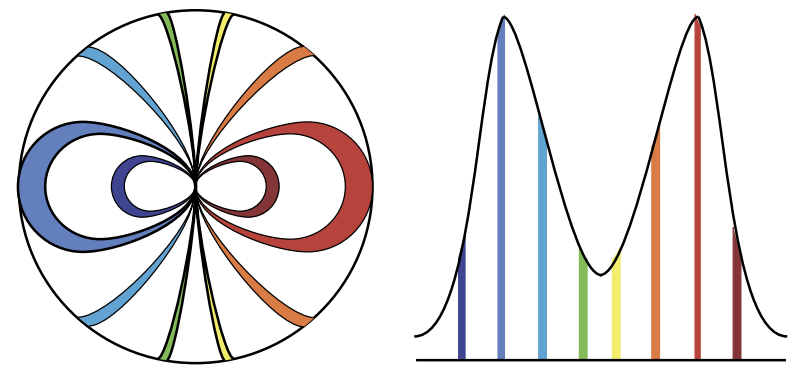

Fig. 13 Contours of constant velocity on a Keplerian disk inclined at an angle $\theta$ to the observer and their contributions in forming the characteristic Keplerian line-profile. Line minima come from the regions of the disk that is dissected by the observer's line-of-sight, while maxima are produced for the longest closed iso-velocity contours which occur at $R_{\text {out }}$ (from Dionatos 2015)

\subsection{Emission-Line Profiles of Disks}

Line broadening from gas following the Keplerian rotation of a disk results in a characteristic double-peaked profile (often coined as the Keplerian profile). This is a characteristic signature that can distinguish emission from gaseous disks from other sources of excited gas (envelope cavities for embedded protostars and jets for accreting disk sources).

The line-of-sight loci of gas moving at a constant velocity on a Keplerian rotating disk at an inclination $\theta$ to the observer are given by the relation (Beckwith and Sargent 1993):

$$
R=R_{\text {out }}\left(\frac{u_{\text {out }}}{u}\right)^{2} \cos ^{2} \theta,
$$

where $u_{\text {out }}$ is the velocity at the terminating radius $R_{\text {out }}$ (Fig. 13). The minimum of the line occurs when the velocity vector of the observer $u \rightarrow 0$ and corresponds to the regions in the disk where the gas velocity is directed perpendicularly to the line-of-sight. As long as the velocity remains smaller than $u_{\text {out }}$, the iso-velocity contours on the disk surface are open and truncated at $R_{\text {out }}$. The maximum contour occurs for $u=u_{\text {out }}$ that is the first closed (nontruncated) contour which contributes to the line peaks. Further increase in the velocity for $u>u_{\text {out }}$ results in shorter, closed contours lying symmetrically on the horizontal disk midline that move towards to the center of the disk and contribute to the outer line wings. The contribution of the different curves of constant velocity to the line profile is illustrated in Fig. 13. The limiting $u_{\text {out }}$ can be derived from the separation of the line-peaks and Eq. (1) once $R_{\text {out }}$ and the inclination $\theta$ are known.

\subsection{New Input from ALMA}

Keplerian profiles are commonly seen in disks of $\mathrm{T}$ Tauri and Herbig Ae/Be stars when observed with adequate angular and spectral resolutions, and indeed Keplerian rotation was already observed in such environments even before the advent of ALMA (e.g. Piétu et al. 2005, 2007; Dutrey et al. 2008). Careful analysis and modeling of spectroimaging observations can lead in constraining the physical conditions of specific layers in the disk traced by molecular lines (e.g. review by Dutrey et al. 2007). Most star formation theories predicted that such disks form early on, but it was only very recently that the first disks displaying Keplerian rotation were observed with ALMA around very young, embedded protostars (Tobin et al. 2012; Takakuwa et al. 2012; Murillo et al. 2013; Ohashi et al. 2014). 
In disks around $\mathrm{T}$ Tauri and Herbig Ae/Be stars, observations of the $\mathrm{CO}$ snowline (Qi et al. 2013, see also Sect. 6.3) have for the first time detected the borderlines that define the conditions for the possible formation of rocky and gas-giant planets. High concentrations of dust observed in a number of transitional disks (e.g. van der Marel et al. 2015; Pérez et al. 2014b) may be the result of the interaction between planets or disk fragments (see Sect. 2.2) with the disk. In these cases, the gas appears to fill the dust cavities but at reduced levels (van der Marel et al. 2015), and in some cases show asymmetric distributions that may be related to the dust asymmetries (e.g. HCN and CS, van der Plas et al. 2014).

Large asymmetries and deviations from Keplerian rotation have also been observed in the distribution of the CO emission around the T Tauri star AS 205 (Salyk et al. 2014). However, in that case the influence of a close companion may drive tidal interactions, or a disk wind may be in action (see Sect. 5.3 and 8), or the combination of both.

An unexpected result comes from the observations of IM Lup in $\mathrm{DCO}^{+}$(Öberg et al. 2015), showing two co-centric rings at radii of $\sim 90$ and 300 au. While the inner ring can be explained in terms of thermal desorption of $\mathrm{CO}$, the outer ring is interpreted in terms of non-thermal desorption of $\mathrm{CO}$ which reacts with $\mathrm{H}_{2} \mathrm{D}^{+}$to form the observed $\mathrm{DCO}^{+}$.

\section{Observational Constraints of Photoevaporative Disk Winds}

\subsection{Atomic Tracers}

Direct observations of photoevaporative disks winds are needed to test and constrain the different models and ultimately characterize them. In this section we will only cover the observational aspects of disk winds, and refer the reader to the Chap. 6 by Gorti et al. for a complete overview of photoevaporation in the context of disk dispersal and to the review by Alexander et al. (2014). A brief overview of the basic theory of photoevaporation is given in the present chapter (see Sect. 5.3).

Forbidden line emission often observed in T Tauri stars can potentially trace photoevaporative disk winds. Neon has high first and second ionization potentials $(21.56$ and $41.0 \mathrm{eV}$, respectively). Ionization can also occur by photoionization of the inner shell, requiring 0.87 and $0.88 \mathrm{keV}$ for ejecting a K-shell electron for neutral and singly ionized neon. Only photons in the EUV range (or beyond) can ionize the outer electrons, while hard X-rays with energies in the range of $\mathrm{keV}$ are needed to eject a K-shell electron. Therefore neon can be used to constrain the high-energy stellar radiation reaching the disk. The mid-infrared forbidden transition of [Ne II] $(12.81 \mu \mathrm{m})$ was first detected with the Spitzer Infrared Spectrograph (IRS, Pascucci et al. 2007, Lahuis et al. 2007). Although the Spitzer spectrograph lacks the spectral resolution needed to confirm or rule out a disk, wind, or jet origin of the line, interesting conclusions were extracted from studies based on statistically significant samples (see for example Lahuis et al. 2007, Flaccomio et al. 2009, Güdel et al. 2010, Baldovin-Saavedra et al. 2011). For example, the line luminosities of sources known to drive outflows and jets are brighter by 1-2 orders of magnitude than those of sources without outflows/jets. Correlations are observed between the line luminosity and different stellar and disk physical parameters, the most prominent ones being with the stellar X-ray luminosity $L_{\mathrm{X}}$ and the disk mass accretion rate.

The [Ne III] line $(15.55 \mu \mathrm{m})$ on the other hand is detected only in a handful of objects (e.g., Kruger et al. 2013, Espaillat et al. 2013). Espaillat et al. (2013) tested the possibility of the lines being emitted by an X-ray and EUV irradiated disk. The study shows no difference in the X-ray properties between stars with and without [Ne III] emission. In addition the 
authors used the ratio [ $\mathrm{Ne} \mathrm{III}] /[\mathrm{Ne} \mathrm{II}]$ to distinguish between X-ray and EUV production of the fine-structure emission. In fact, if the emission is dominated by X-ray production the ratio is expected to be $\sim 0.1$; in the case of EUV the line ratio can be much larger. These authors observed one object for which the ratio is $\sim 1$ and therefore consistent with EUV production. However, it is not possible to make general conclusions given the small number of detections.

A natural step in the study of [Ne II] emission was to follow-up with ground-based observations those stars that display [Ne II] emission in their Spitzer spectra. Thanks to the high-spectral resolution achievable with ground-based spectrographs in the mid-infrared ( $R \sim 30000$, or $10 \mathrm{~km} \mathrm{~s}^{-1}$ for VISIR at VLT), lines could be spectrally resolved, and the nature of the emission (disk, disk wind, or jet) for most of the objects could finally be unveiled. At the time of writing the neon line has been observed with ground-based instruments in $\sim 50$ objects (see Herczeg et al. 2007, Najita et al. 2009, Pascucci and Sterzik 2009, van Boekel et al. 2009, Pascucci et al. 2011, Sacco et al. 2012, Baldovin-Saavedra et al. 2012). About $50 \%$ of the [Ne II] lines detected with Spitzer are confirmed with ground based observations. This hints at a high contribution from shocked material in the Spitzer spectra. The lines that are interpreted as emitted in jets present high-velocity blueshifts $\left(30 \mathrm{~km} \mathrm{~s}^{-1}\right.$ or more) and their full width at half maximum (FWHM) can be quite broad (FWHM up to $\sim 140 \mathrm{~km} \mathrm{~s}^{-1}$ ). Lines consistent with disk wind emission show small velocity blueshifts $\left(\sim 2-20 \mathrm{~km} \mathrm{~s}^{-1}\right)$ and are narrow (FWHM $\sim 10-25 \mathrm{~km} \mathrm{~s}^{-1}$ ). There is a handful of objects that show no shift in their line peak. However, they cannot be directly associated to disk emission; two of them (AA Tau, CoKu Tau 1) are high inclination objects known to drive bipolar jets. An interesting result from these studies is that the [Ne II] line shows only one velocity component, either a high-velocity jet, a disk wind, or a disk atmosphere. There is no evidence up to now of a neon line with two velocity components as is often observed in other atomic tracers such as [O I] in the optical.

Forbidden lines in the optical range are common in T Tauri stars and have been the subject of studies for quite some time (Jankovics et al. 1983, Edwards et al. 1987, Hamann 1994, Hartigan et al. 1995). Early studies reported the presence of blueshifted line emission and associated it with some sort of disk wind whose redshifted emission is obscured by the protoplanetary disk. Forbidden emission lines often display two-velocity components: a high-velocity component (HVC) blueshifted by hundred kilometers per second with respect to the stellar rest velocity, and a low-velocity component (LVC) blueshifted by a few kilometers per second. Kwan and Tademaru (1988) recognized that these two components are emitted in two different regions and attributed the low-velocity component to a slow-moving wind emitted from the disk, and the high-velocity component to a collimated jet near the star. In addition, Hartigan et al. (1995) observed a trend where the line velocity shifts are higher for lower critical densities, suggesting that the low-velocity component accelerates away from the surface of the disk.

The neutral atomic oxygen lines [O I] at $6300 \AA$ and [O I] at $5577 \AA$ are interesting because they are typically bright, in particular the $6300 \AA$ line. Different authors have attempted to explain the observed properties of the [O I] line with thermal photoevaporative disk wind models considering different types of stellar radiation. The model presented in Font et al. (2004), where photoevaporation is driven by stellar EUV radiation, is difficult to reconcile with the observed [O I] line fluxes because it produces an almost fully ionized layer with a low fraction of neutral gas. Ercolano and Owen (2010) modeled photoevaporation driven by EUV and soft X-ray emission (0.1-10 keV) that can heat the gas to the high temperatures needed to reproduce the observed [O I] luminosity. Gorti et al. (2011) considered FUV and X-rays in their input stellar spectrum and found that the [O I] lines are emitted from dissociation of $\mathrm{OH}$ with only a minor contribution from thermal emission. 


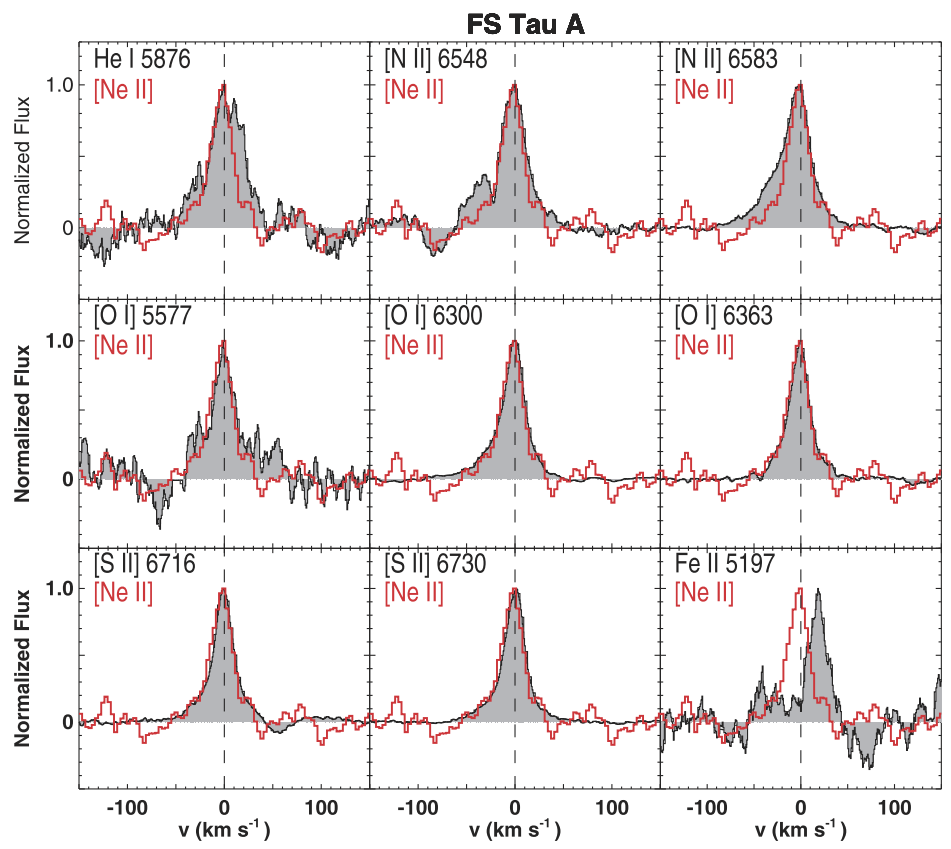

Fig. 14 Comparison between the [Ne II] line $(12.81 \mu \mathrm{m})$ detected with VISIR (VLT) and the optical lines detected with UVES (VLT) in the young system FS Tau A. In this case the line centroids of the mid-infrared tracer are in good agreement with the optical forbidden lines (Baldovin-Saavedra et al. 2012, CESO/A\&A. Reproduced with permission.)

Recent observational studies have focused on the study of optical forbidden lines (Baldovin-Saavedra et al. 2012, Rigliaco et al. 2013, Natta et al. 2014, Manara et al. 2014). Baldovin-Saavedra et al. (2012) obtained UVES (VLT) high-resolution spectra for three objects with a [Ne II] line detected from the ground. In two of the objects the optical and the mid-infrared tracers display the same velocity shift and FWHM (see Fig. 14), and in one case the [Ne II] line traces clearly jet emission, while the [O I] lines show two velocity components. Rigliaco et al. (2013) performed a systematic study of the [O I] low-velocity component and compared it to the $[\mathrm{Ne} \mathrm{II}]$ and $\mathrm{CO}$ lines. They find a trend between the line peak velocities, where the $[\mathrm{Ne} \mathrm{II}]$ line is typically more blueshifted than $[\mathrm{O} \mathrm{I}]$ which in turn is more blueshifted than $\mathrm{CO}$. The authors obtained a small range in the line ratio of [O I] 6300/5577 $\AA$ which they claimed is difficult to reproduce in gas heated by thermal processes. For two objects observed at very high spectral resolution the authors could decompose the line profile in two components: a broad and symmetric component attributed to disk emission and a narrower and blueshifted component attributed to unbound gas. Natta et al. (2014) found a correlation between the [O I] luminosity and both $L_{\star}$ and $L_{\text {acc }}$, the latter being related to the stellar FUV emission. They found that, on average, the low-velocity component peaks at $<20 \mathrm{~km} \mathrm{~s}^{-1}$ and determined the physical conditions of the gas, finding high density $\left(n_{\mathrm{H}}>10^{8} \mathrm{~cm}^{-3}\right)$ and temperatures $(T \sim 5000-1000 \mathrm{~K})$. The authors warn that the absence of correlation between the luminosity of the [O I] line and $L_{\mathrm{X}}$, contradicts an $\mathrm{X}$-ray driven disk wind. 
Fig. 15 The grey area represents the $\mathrm{H}_{2} 1-0 \mathrm{~S}(1)$ line at $2.12 \mu \mathrm{m}$ detected with CRIRES and the solid line represents the [Ne II] line $(12.81 \mu \mathrm{m})$ detected with VISIR in FS Tau A (Baldovin-Saavedra et al. in prep.)

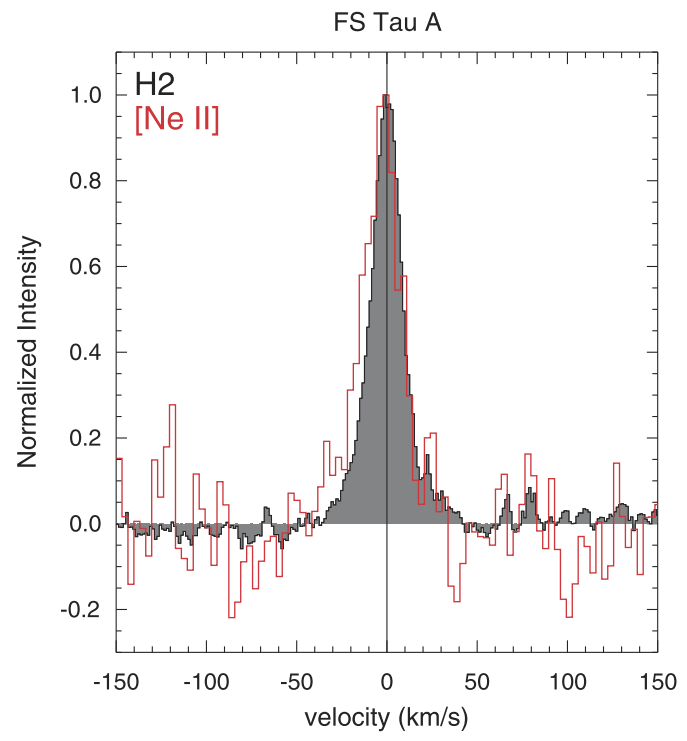

\subsection{Molecular Tracers}

$\mathrm{CO}$ ro-vibrational emission at $4.7 \mu \mathrm{m}$ is detected in many $\mathrm{T}$ Tauri stars. The line probes warm gas in the inner $1 \mathrm{au}$ from the central star. Some of the observed lines show a singly peaked and narrow profile with a broad base that extends to $>50 \mathrm{~km} \mathrm{~s}^{-1}$; they show a small velocity shift of $\sim 5 \mathrm{~km} \mathrm{~s}^{-1}$ that cannot be explained by purely Keplerian rotation (e.g., Pontoppidan et al. 2011, Bast et al. 2011, Brown et al. 2013). Pontoppidan et al. (2011) performed spectroastrometry observations of the $\mathrm{CO}$ line. This technique allows to obtain spatial and kinematical information at very small scales and very high spectral resolution. In their study, they find that the single-peaked line profiles are consistent with a combination of gas in Keplerian rotation and a non-collimated disk wind. In addition, Brown et al. (2013) detected excess emission on the blue side of the line profile, giving further confirmation for the disk wind hypothesis.

Molecular hydrogen, $\mathrm{H}_{2}$, is the most abundant component in protoplanetary disks, but this homonuclear molecule is very difficult to observe since its rotational quadrupole transitions from low-energy levels are weak and lie in regions with poor atmospheric transmission. The ro-vibrational transitions observable in the near-infrared trace thermal emission of hot gas at about $1000 \mathrm{~K}$, or gas excited by UV or X-rays (Carmona et al. 2007, 2011). Takami et al. (2004) reported the detection of a slow molecular wind in $\mathrm{H}_{2}$ for DG Tau. BaldovinSaavedra et al. (in prep.) performed follow-up observations with CRIRES (VLT) of young systems with detections of [Ne II]. The program aimed at detecting the ro-vibrational transitions of $\mathrm{H}_{2}: 1-0 \mathrm{~S}(1)(2.12 \mu \mathrm{m}), 1-0 \mathrm{~S}(1)(2.22 \mu \mathrm{m})$, and 2-1 S(1) $(2.25 \mu \mathrm{m})$. Emission from the three transitions is detected in three objects, two of them known to drive highvelocity jets. Two of the sources have line profiles consistent with emission from the disk atmosphere (no shift), and one shows a blueshift of $\sim-8 \mathrm{~km} \mathrm{~s}^{-1}$. The other four objects show detections from the 1-0 S(0) transition only; two of them can be interpreted in terms of gas bound to the disk, and two present lines with small blueshifts $\left(<-10 \mathrm{~km} \mathrm{~s}^{-1}\right)$. Figure 15 (Baldovin-Saavedra et al. in prep.) shows a comparison between the $\mathrm{H}_{2} 1-0 \mathrm{~S}$ (1) line and the $[\mathrm{Ne}$ II] line detected in the mid-infrared and published in Baldovin-Saavedra et al. 
(2012) for one of the stars in the sample. The spectra have been continuum subtracted and the lines normalized to their peak to allow for a better comparison. In this case the [Ne II] line is slightly blueshifted, $v_{\text {peak }}=-2.9 \pm 0.7 \mathrm{~km} \mathrm{~s}^{-1}$ with a FWHM of $26.8 \mathrm{~km} \mathrm{~s}^{-1}$ and shows excess emission towards the blue side of the spectrum, while the $\mathrm{H}_{2}$ line peak is consistent with disk emission, $v_{\text {peak }}=-0.1 \pm 0.2 \mathrm{~km} \mathrm{~s}^{-1}$ and FWHM $=21.2 \pm 0.2 \mathrm{~km} \mathrm{~s}^{-1}$. It is interesting to estimate the distance from the star needed to observe a line in Keplerian rotation that does not show a double peaked profile. Considering the inclination of the system, $i=35^{\circ}$, the mass of the star $M_{\star}=0.6 \mathrm{M}_{\odot}$, and the CRIRES spectral resolution of $3 \mathrm{~km} \mathrm{~s}^{-1}$, the gas should be located at a distance $R>77$ au.

The trend observed in $\mathrm{H}_{2}$ is similar to what is observed in $\mathrm{CO}$; the molecular lines tend to be less blueshifted than the [Ne II] line. However, at this stage it is difficult to establish a direct relation between the observed [Ne II] photoevaporative disk winds and their molecular counterpart. Furthermore, the nature of the molecular winds, i.e., magnetically driven or photoevaporation driven, remain uncertain.

\section{Summary}

We discussed several physical processes which have significant impact on the evolution and the chemical composition of the gas disk. In early stages the disk evolution might be dominated by gravitational instabilities if the initial gas mass is high enough. At this stage most disks probably experience phases of highly increased accretion rates resulting in strong luminosity outbursts. In later phases the disk evolution is dominated by viscous accretion possibly driven by magneto-rotational instabilities. In addition disk winds may play a crucial role in the dispersal of the disk determining the time-scale for planet formation. One example are photo-evaporative winds which are driven by high energy stellar radiation of the star (see also Chap. 6 by Gorti et al.).

Chemical processes in the gas disks determine the chemical composition of planetary atmospheres but also have an impact on the solids due to freeze-out of molecules. Enhanced dust growth at the position of ice lines leads to the formation of larger solid bodies which possibly grow further to planetesimals and planetary cores. The gas mass is another crucial parameter for planet formation. It is still challenging to measure directly the disk gas mass. Using the $\mathrm{CO}$ molecular line emission requires detailed chemical models (e.g. CO isotopologue chemistry and surface chemistry) and therefore the derived masses are still model dependent. The HD molecule offers a more direct method but is currently only detected in one disk. Upcoming missions like the James Webb Space Telescope will certainly improve this situation.

The high spatial resolution and sensitivity of ALMA provides detailed observations of the chemical composition of disks (e.g. detection of complex molecules, ice line locations). Future ALMA observations will certainly significantly improve our knowledge of disk chemistry. Interpretations of these observations are a formidable challenge for chemical models. Models consistently treating the dust and gas evolution are necessary. The incorporation of chemical processes in hydrodynamical models is required for a better understanding of the time evolution of the gaseous component of disks. Further detailed knowledge of the evolution of young stars is required as in particular high-energy stellar radiation is one of the main drivers of disk chemistry and evolution.

Acknowledgements We want to thank the anonymous referee for a thorough and constructive report. We are grateful to the organizers of this International Space Science Institute (ISSI) workshop and the editors of this book. C. Rab and M. Güdel acknowledge funding by the Austrian Science Fund (FWF), project 
number P24790 and project S11601-N16 "Pathways to Habitability: From Disks to Active Stars, Planets and Life". C. Baldovin-Saavedra acknowledges funding by the Austrian Research Promotion Agency (FFG) under grant agreement FA 538022. E. Vorobyov acknowledges support from the RFBR grant 14-02-00719. The research leading to these results has received funding from the European Union Seventh Framework Programme FP7-2011 under grant agreement no 284405. This work was partly supported by the Austrian Science Fund (FWF) under research grant I2549-N27. This publication was partly supported from the FFG ASAP 12 project JetPro* (FFG-854025). The computational results presented have been achieved in part using the Vienna Scientific Cluster (VSC).

Open Access This article is distributed under the terms of the Creative Commons Attribution 4.0 International License (http://creativecommons.org/licenses/by/4.0/), which permits unrestricted use, distribution, and reproduction in any medium, provided you give appropriate credit to the original author(s) and the source, provide a link to the Creative Commons license, and indicate if changes were made.

\section{References}

Y. Aikawa, T. Umebayashi, T. Nakano, S. Miyama, Faraday Discuss. 109, 281 (1998)

Y. Aikawa, G.J. van Zadelhoff, E.F. van Dishoeck, E. Herbst, Astron. Astrophys. 386, 622-632 (2002)

Y. Aikawa, K. Furuya, H. Nomura, C. Qi, Astrophys. J. 807, 120 (2015)

V. Akimkin, S. Zhukovska, D. Wiebe, D. Semenov, Y. Pavlyuchenkov, A. Vasyunin, T. Birnstiel, T. Henning, Astrophys. J. 766, 8 (2013)

R.D. Alexander, C.J. Clarke, J.E. Pringle, Mon. Not. R. Astron. Soc. 369, 216-228 (2006a)

R.D. Alexander, C.J. Clarke, J.E. Pringle, Mon. Not. R. Astron. Soc. 369, 229-239 (2006b)

R. Alexander, I. Pascucci, S. Andrews, P. Armitage, L. Cieza, in Protostars and Planets VI (2014), pp. 475496

ALMA Partnership, C.L. Brogan, L.M. Pérez, T.R. Hunter, W.R.F. Dent, A.S. Hales, R.E. Hills, S. Corder, E.B. Fomalont, C. Vlahakis, Y. Asaki, D. Barkats, A. Hirota, J.A. Hodge, C.M.V. Impellizzeri, R. Kneissl, E. Liuzzo, R. Lucas, N. Marcelino, S. Matsushita, K. Nakanishi, N. Phillips, A.M.S. Richards, I. Toledo, R. Aladro, D. Broguiere, J.R. Cortes, P.C. Cortes, D. Espada, F. Galarza, D. Garcia-Appadoo, L. Guzman-Ramirez, E.M. Humphreys, T. Jung, S. Kameno, R.A. Laing, S. Leon, G. Marconi, A. Mignano, B. Nikolic, L.-A. Nyman, M. Radiszcz, A. Remijan, J.A. Rodón, T. Sawada, S. Takahashi, R.P.J. Tilanus, B. Vila Vilaro, L.C. Watson, T. Wiklind, E. Akiyama, E. Chapillon, I. de Gregorio-Monsalvo, J. Di Francesco, F. Gueth, A. Kawamura, C.-F. Lee, Q. Nguyen Luong, J. Mangum, V. Pietu, P. Sanhueza, K. Saigo, S. Takakuwa, C. Ubach, T. van Kempen, A. Wootten, A. Castro-Carrizo, H. Francke, J. Gallardo, J. Garcia, S. Gonzalez, T. Hill, T. Kaminski, Y. Kurono, H.-Y. Liu, C. Lopez, F. Morales, K. Plarre, G. Schieven, L. Testi, L. Videla, E. Villard, P. Andreani, J.E. Hibbard, K. Tatematsu, Astrophys. J. Lett. 808, 3 (2015)

P. André, D. Ward-Thompson, J. Greaves, Science 337, 69 (2012)

S.M. Andrews, J.P. Williams, Astrophys. J. 631, 1134-1160 (2005)

S.M. Andrews, D.J. Wilner, C. Espaillat, A.M. Hughes, C.P. Dullemond, M.K. McClure, C. Qi, J.M. Brown, Astrophys. J. 732, 42 (2011)

S.M. Andrews, D.J. Wilner, Z. Zhu, T. Birnstiel, J.M. Carpenter, L.M. Pérez, X.-N. Bai, K.I. Öberg, A.M. Hughes, A. Isella, L. Ricci, Astrophys. J. Lett. 820, 40 (2016)

G. Aresu, I. Kamp, R. Meijerink, P. Woitke, W.-F. Thi, M. Spaans, Astron. Astrophys. 526, 163 (2011)

G. Aresu, I. Kamp, R. Meijerink, M. Spaans, S. Vicente, L. Podio, P. Woitke, F. Menard, W.-F. Thi, M. Güdel, A. Liebhart, Astron. Astrophys. 566, 14 (2014)

M. Audard, P. Ábrahám, M.M. Dunham, J.D. Green, N. Grosso, K. Hamaguchi, J.H. Kastner, Á. Kóspál, G. Lodato, M.M. Romanova, S.L. Skinner, E.I. Vorobyov, Z. Zhu, in Protostars and Planets VI (2014), pp. $387-410$

S.A. Balbus, J.F. Hawley, Astrophys. J. 376, 214-233 (1991)

C. Baldovin-Saavedra, M. Audard, M. Güdel, L.M. Rebull, D.L. Padgett, S.L. Skinner, A. Carmona, A.M. Glauser, S.B. Fajardo-Acosta, Astron. Astrophys. 528, 22 (2011)

C. Baldovin-Saavedra, M. Audard, A. Carmona, M. Güdel, K. Briggs, L.M. Rebull, S.L. Skinner, B. Ercolano, Astron. Astrophys. 543, 30 (2012)

J. Bally, E. Feigelson, B. Reipurth, Astrophys. J. 584, 843-852 (2003)

C. Baruteau, A. Crida, S.-J. Paardekooper, F. Masset, J. Guilet, B. Bitsch, R. Nelson, W. Kley, J. Papaloizou, in Protostars and Planets VI (2014), pp. 667-689

J.E. Bast, J.M. Brown, G.J. Herczeg, E.F. van Dishoeck, K.M. Pontoppidan, Astron. Astrophys. 527, 119 (2011) 
S. Basu, E.I. Vorobyov, Astrophys. J. 750, 30 (2012)

M.R. Bate, Mon. Not. R. Astron. Soc. 392, 590-616 (2009)

S.V.W. Beckwith, A.I. Sargent, Astrophys. J. 402, 280-291 (1993)

E.A. Bergin, Y. Aikawa, G.A. Blake, E.F. van Dishoeck, in Protostars and Planets V (2007), pp. 751-766. http://arxiv.org/abs/astro-ph/0603358

E.A. Bergin, L.I. Cleeves, U. Gorti, K. Zhang, G.A. Blake, J.D. Green, S.M. Andrews, N.J. Evans II, T. Henning, K. Öberg, K. Pontoppidan, C. Qi, C. Salyk, E.F. van Dishoeck, Nature 493, 644-646 (2013)

E.A. Bergin, L.I. Cleeves, N. Crockett, G.A. Blake, Faraday Discuss. 168, 61-79 (2014). http://adsabs. harvard.edu/abs/2014FaDi..168...61B

B. Bitsch, A. Johansen, M. Lambrechts, A. Morbidelli, Astron. Astrophys. 575, 28 (2015)

A.C. Boley, Astrophys. J. Lett. 695, 53-57 (2009)

A.C. Boley, T. Hayfield, L. Mayer, R.H. Durisen, Icarus 207, 509-516 (2010)

A.P. Boss, Science 276, 1836-1839 (1997)

A.P. Boss, Astrophys. J. 731, 74 (2011)

J.M. Brown, K.M. Pontoppidan, E.F. van Dishoeck, G.J. Herczeg, G.A. Blake, A. Smette, Astrophys. J. 770, 94 (2013)

S. Bruderer, E.F. van Dishoeck, S.D. Doty, G.J. Herczeg, Astron. Astrophys. 541, 91 (2012)

J.R. Burke, D.J. Hollenbach, Astrophys. J. 265, 223-234 (1983)

S. Cabrit, constraints on accretion-ejection structures in young stars, in EAS Publications Series, ed. by J. Bouvier, J.-P. Zahn, EAS Publications Series, vol. 3, 2002, pp. 147-182

N. Calvet, E. Gullbring, Astrophys. J. 509, 802-818 (1998)

A. Carmona, Earth Moon Planets 106, 71-95 (2010)

A. Carmona, M.E. van den Ancker, T. Henning, M. Goto, D. Fedele, B. Stecklum, Astron. Astrophys. 476, 853 (2007). doi:10.1051/0004-6361:20078536. Erratum: 478, 795 (2008)

A. Carmona, G. van der Plas, M.E. van den Ancker, M. Audard, L.B.F.M. Waters, D. Fedele, B. Acke, E. Pantin, Astron. Astrophys. 533, 39 (2011)

C. Ceccarelli, C. Dominik, A. López-Sepulcre, M. Kama, M. Padovani, E. Caux, P. Caselli, Astrophys. J. Lett. 790, 1 (2014)

G. Chaparro Molano, I. Kamp, Astron. Astrophys. 537, 138 (2012a)

G. Chaparro Molano, I. Kamp, Astron. Astrophys. 547, 7 (2012b)

E.I. Chiang, P. Goldreich, Astrophys. J. 490, 368 (1997)

C.J. Clarke, A. Gendrin, M. Sotomayor, Mon. Not. R. Astron. Soc. 328, 485-491 (2001)

L.I. Cleeves, F.C. Adams, E.A. Bergin, Astrophys. J. 772, 5 (2013a)

L.I. Cleeves, F.C. Adams, E.A. Bergin, R. Visser, Astrophys. J. 777, 28 (2013b)

L.I. Cleeves, E.A. Bergin, C. Qi, F.C. Adams, K.I. Öberg, Astrophys. J. 799, 204 (2015)

O. Dionatos, Gas line observations of disks. Eur. Phys. J. Web Conf. 102, 00008 (2015)

F. Du, E.A. Bergin, Astrophys. J. 792, 2 (2014)

M.M. Dunham, E.I. Vorobyov, Astrophys. J. 747, 52 (2012)

M.M. Dunham, E.I. Vorobyov, H.G. Arce, Mon. Not. R. Astron. Soc. 444, 887-901 (2014)

M.M. Dunham, N.J. Evans II, S. Terebey, C.P. Dullemond, C.H. Young, Astrophys. J. 710, 470-502 (2010)

A. Dutrey, S. Guilloteau, P. Ho, in Protostars and Planets V (2007), pp. 495-506

A. Dutrey, S. Guilloteau, V. Piétu, E. Chapillon, F. Gueth, T. Henning, R. Launhardt, Y. Pavlyuchenkov, K. Schreyer, D. Semenov, Astron. Astrophys. 490, 15-18 (2008)

A. Dutrey, D. Semenov, E. Chapillon, U. Gorti, S. Guilloteau, F. Hersant, M. Hogerheijde, M. Hughes, G. Meeus, H. Nomura, V. Piétu, C. Qi, V. Wakelam, in Protostars and Planets VI (2014), pp. 317-338

S. Edwards, S. Cabrit, S.E. Strom, I. Heyer, K.M. Strom, E. Anderson, Astrophys. J. 321, 473-495 (1987)

B. Ercolano, J.E. Owen, Mon. Not. R. Astron. Soc. 406, 1553-1569 (2010)

B. Ercolano, G. Rosotti, Mon. Not. R. Astron. Soc. 450, 3008-3014 (2015)

B. Ercolano, J.J. Drake, J.C. Raymond, C.C. Clarke, Astrophys. J. 688, 398-407 (2008)

C. Espaillat, L. Ingleby, E. Furlan, M. McClure, A. Spatzier, J. Nieusma, N. Calvet, E. Bergin, L. Hartmann, J.M. Miller, J. Muzerolle, Astrophys. J. 762, 62 (2013)

C. Espaillat, J. Muzerolle, J. Najita, S. Andrews, Z. Zhu, N. Calvet, S. Kraus, J. Hashimoto, A. Kraus, P. D'Alessio, in Protostars and Planets VI (2014), pp. 497-520

M.G. Evans, J.D. Ilee, A.C. Boley, P. Caselli, R.H. Durisen, T.W. Hartquist, J.M.C. Rawlings, Mon. Not. R. Astron. Soc. 453, 1147-1163 (2015)

F. Favata, C.V.M. Fridlund, G. Micela, S. Sciortino, A.A. Kaas, Astron. Astrophys. 386, 204-210 (2002)

F. Favata, G. Micela, B. Silva, S. Sciortino, M. Tsujimoto, Astron. Astrophys. 433, 1047-1054 (2005)

C. Favre, L.I. Cleeves, E.A. Bergin, C. Qi, G.A. Blake, Astrophys. J. Lett. 776, 38 (2013)

D. Fedele, M.E. van den Ancker, T. Henning, R. Jayawardhana, J.M. Oliveira, Astron. Astrophys. 510, 72 (2010)

E.D. Feigelson, G.P. Garmire, S.H. Pravdo, Astrophys. J. 572, 335-349 (2002) 
E. Flaccomio, B. Stelzer, S. Sciortino, G. Micela, I. Pillitteri, L. Testi, Astron. Astrophys. 505, 695-706 (2009)

A.S. Font, I.G. McCarthy, D. Johnstone, D.R. Ballantyne, Astrophys. J. 607, 890-903 (2004)

A. Frank, T.P. Ray, S. Cabrit, P. Hartigan, H.G. Arce, F. Bacciotti, J. Bally, M. Benisty, J. Eislöffel, M. Güdel, S. Lebedev, B. Nisini, A. Raga, in Protostars and Planets VI (2014), pp. 451-474

S. Fromang, C. Terquem, S.A. Balbus, Mon. Not. R. Astron. Soc. 329, 18-28 (2002)

K. Furuya, Y. Aikawa, Astrophys. J. 790, 97 (2014)

C.F. Gammie, Astrophys. J. 457, 355 (1996)

P.G. Gibbons, G.R. Mamatsashvili, W.K.M. Rice, Mon. Not. R. Astron. Soc. 453, 4232-4243 (2015)

A.E. Glassgold, D. Galli, M. Padovani, Astrophys. J. 756, 157 (2012)

A.E. Glassgold, J. Najita, J. Igea, Astrophys. J. 480, 344-350 (1997)

A.E. Glassgold, J. Najita, J. Igea, Astrophys. J. 615, 972-990 (2004)

U. Gorti, D. Hollenbach, Astrophys. J. 613, 424-447 (2004)

U. Gorti, D. Hollenbach, Astrophys. J. 683, 287-303 (2008)

U. Gorti, D. Hollenbach, J. Najita, I. Pascucci, Astrophys. J. 735, 90 (2011)

R. Gredel, S. Lepp, A. Dalgarno, E. Herbst, Astrophys. J. 347, 289-293 (1989)

M. Güdel, Annu. Rev. Astron. Astrophys. 12, 71-237 (2004)

M. Güdel, A. Telleschi, Astron. Astrophys. 474, 25-28 (2007)

M. Güdel, S.L. Skinner, M. Audard, K.R. Briggs, S. Cabrit, Astron. Astrophys. 478, 797-807 (2008)

M. Güdel, F. Lahuis, K.R. Briggs, J. Carr, A.E. Glassgold, T. Henning, J.R. Najita, R. van Boekel, E.F. van Dishoeck, Astron. Astrophys. 519, 113 (2010)

F. Hamann, Astrophys. J. Suppl. Ser. 93, 485-518 (1994)

N. Harada, E. Herbst, V. Wakelam, Astrophys. J. 721, 1570-1578 (2010)

P. Hartigan, S. Edwards, L. Ghandour, Astrophys. J. 452, 736 (1995)

C. Helling, P. Woitke, P.B. Rimmer, I. Kamp, W.-F. Thi, R. Meijerink, Life 4(2), 142-173 (2014)

T. Henning, D. Semenov, Chem. Rev. 113, 9016-9042 (2013)

G.J. Herczeg, J.R. Najita, L.A. Hillenbrand, I. Pascucci, Astrophys. J. 670, 509-515 (2007)

J. Hernández, L. Hartmann, N. Calvet, R.D. Jeffries, R. Gutermuth, J. Muzerolle, J. Stauffer, Astrophys. J. 686, 1195-1208 (2008)

D.J. Hollenbach, A.G.G.M. Tielens, Rev. Mod. Phys. 71, 173-230 (1999)

D. Hollenbach, D. Johnstone, S. Lizano, F. Shu, Astrophys. J. 428, 654-669 (1994)

J. Igea, A.E. Glassgold, Astrophys. J. 518, 848-858 (1999)

J.D. Ilee, A.C. Boley, P. Caselli, R.H. Durisen, T.W. Hartquist, J.M.C. Rawlings, Mon. Not. R. Astron. Soc. 417, 2950-2961 (2011)

K. Imanishi, K. Koyama, Y. Tsuboi, Astrophys. J. 557, 747-760 (2001)

I. Jankovics, I. Appenzeller, J. Krautter, Publ. Astron. Soc. Pac. 95, 883-885 (1983)

J.K. Jørgensen, R. Visser, N. Sakai, E.A. Bergin, C. Brinch, D. Harsono, J.E. Lindberg, E.F. van Dishoeck, S. Yamamoto, S.E. Bisschop, M.V. Persson, Astrophys. J. Lett. 779, 22 (2013)

I. Kamp, G.-J. van Zadelhoff, Astron. Astrophys. 373, 641-656 (2001)

I. Kamp, I. Tilling, P. Woitke, W. Thi, M. Hogerheijde, Astron. Astrophys. 510(26), 260000 (2010)

J.H. Kastner, D.P. Huenemoerder, N.S. Schulz, C.R. Canizares, D.A. Weintraub, Astrophys. J. 567, 434-440 (2002)

S.J. Kenyon, L.W. Hartmann, K.M. Strom, S.E. Strom, Astron. J. 99, 869-887 (1990)

H.J. Kim, N.J. Evans II, M.M. Dunham, J.-E. Lee, K.M. Pontoppidan, Astrophys. J. 758, 38 (2012)

K.M. Kratter, R.A. Murray-Clay, Astrophys. J. 740, 1 (2011)

K.M. Kratter, C.D. Matzner, M.R. Krumholz, Astrophys. J. 681, 375-390 (2008)

A.J. Kruger, M.J. Richter, J.S. Carr, J.R. Najita, M.M. Moerchen, G.W. Doppmann, A. Seifahrt, Astrophys. J. 764, 127 (2013)

J. Kwan, E. Tademaru, Astrophys. J. 332, L41-L44 (1988). doi:10.1086/185262

F. Lahuis, E.F. van Dishoeck, G.A. Blake, N.J. Evans II, J.E. Kessler-Silacci, K.M. Pontoppidan, Astrophys. J. 665, 492-511 (2007)

J.-E. Lee, J. Korean Astron. Soc. 40, 83-89 (2007)

Z.-Y. Li, R. Banerjee, R.E. Pudritz, J.K. Jørgensen, H. Shang, R. Krasnopolsky, A. Maury, in Protostars and Planets VI (2014), pp. 173-194

M.N. Machida, S.-i. Inutsuka, T. Matsumoto, Astrophys. J. 724, 1006-1020 (2010)

M.N. Machida, S.-i. Inutsuka, T. Matsumoto, Astrophys. J. 729, 42 (2011)

E.E. Mamajek, Initial conditions of planet formation: lifetimes of primordial disks, in American Institute of Physics Conference Series, ed. by T. Usuda, M. Tamura, M. Ishii, American Institute of Physics Conference Series, vol. 1158 (2009), pp. 3-10

C.F. Manara, L. Testi, A. Natta, G. Rosotti, M. Benisty, B. Ercolano, L. Ricci, Astron. Astrophys. 568, 18 (2014) 
B.C. Matthews, A.V. Krivov, M.C. Wyatt, G. Bryden, C. Eiroa, in Protostars and Planets VI (2014), pp. 521544

D. McElroy, C. Walsh, A.J. Markwick, M.A. Cordiner, K. Smith, T.J. Millar, Astron. Astrophys. 550, 36 (2013)

R. Meijerink, G. Aresu, I. Kamp, M. Spaans, W.-F. Thi, P. Woitke, Astron. Astrophys. 547, 68 (2012)

F. Meru, M.R. Bate, Mon. Not. R. Astron. Soc. 427, 2022-2046 (2012)

A. Miotello, S. Bruderer, E.F. van Dishoeck, Astron. Astrophys. 572, 96 (2014)

N.M. Murillo, S.-P. Lai, S. Bruderer, D. Harsono, E.F. van Dishoeck, Astron. Astrophys. 560, 103 (2013)

J.R. Najita, J.S. Carr, A.E. Glassgold, J.A. Valenti, in Protostars and Planets V (2007), pp. 507-522

J.R. Najita, G.W. Doppmann, M.A. Bitner, M.J. Richter, J.H. Lacy, D.T. Jaffe, J.S. Carr, R. Meijerink, G.A. Blake, G.J. Herczeg, A.E. Glassgold, Astrophys. J. 697, 957-963 (2009)

A. Natta, L. Testi, J.M. Alcalá, E. Rigliaco, E. Covino, B. Stelzer, V. D’Elia, Astron. Astrophys. 569, 5 (2014)

S. Nayakshin, Mon. Not. R. Astron. Soc. 408, 36-40 (2010)

S. Nayakshin, Mon. Not. R. Astron. Soc. 413, 1462-1478 (2011)

S. Nayakshin, S.-H. Cha, J.C. Bridges, Mon. Not. R. Astron. Soc. 416, 50-54 (2011)

H. Nomura, T.J. Millar, Astron. Astrophys. 438, 923-938 (2005)

K.I. Öberg, C. Qi, J.K.J. Fogel, E.A. Bergin, S.M. Andrews, C. Espaillat, D.J. Wilner, I. Pascucci, J.H. Kastner, Astrophys. J. 734, 98 (2011)

K.I. Öberg, K. Furuya, R. Loomis, Y. Aikawa, S.M. Andrews, C. Qi, E.F. van Dishoeck, D.J. Wilner, Astrophys. J. 810, 112 (2015)

N. Ohashi, K. Saigo, Y. Aso, Y. Aikawa, S. Koyamatsu, M.N. Machida, M. Saito, S.Z. Takahashi, S. Takakuwa, K. Tomida, K. Tomisaka, H.-W. Yen, Astrophys. J. 796, 131 (2014)

J.E. Owen, B. Ercolano, C.J. Clarke, Mon. Not. R. Astron. Soc. 412, 13-25 (2011)

J.E. Owen, B. Ercolano, C.J. Clarke, R.D. Alexander, Mon. Not. R. Astron. Soc. 401, 1415-1428 (2010)

M. Padovani, P. Hennebelle, A. Marcowith, K. Ferrière, Astron. Astrophys. 582, 13 (2015)

A. Palau, L.A. Zapata, L.F. Rodríguez, H. Bouy, D. Barrado, M. Morales-Calderón, P.C. Myers, N. Chapman, C. Juárez, D. Li, Mon. Not. R. Astron. Soc. 444, 833-845 (2014)

I. Pascucci, M. Sterzik, Astrophys. J. 702, 724-732 (2009)

I. Pascucci, D. Hollenbach, J. Najita, J. Muzerolle, U. Gorti, G.J. Herczeg, L.A. Hillenbrand, J.S. Kim, J.M. Carpenter, M.R. Meyer, E.E. Mamajek, J. Bouwman, Astrophys. J. 663, 383-393 (2007)

I. Pascucci, M. Sterzik, R.D. Alexander, S.H.P. Alencar, U. Gorti, D. Hollenbach, J. Owen, B. Ercolano, S. Edwards, Astrophys. J. 736, 13 (2011)

I. Pascucci, L. Ricci, U. Gorti, D. Hollenbach, N.P. Hendler, K.J. Brooks, Y. Contreras, Astrophys. J. 795, 1 (2014)

L.M. Pérez, A. Isella, J.M. Carpenter, C.J. Chandler, Astrophys. J. Lett. 783, 13 (2014a)

L.M. Pérez, A. Isella, J.M. Carpenter, C.J. Chandler, Astrophys. J. Lett. 783, 13 (2014b)

V. Piétu, A. Dutrey, S. Guilloteau, Astron. Astrophys. 467, 163-178 (2007)

V. Piétu, S. Guilloteau, A. Dutrey, Astron. Astrophys. 443, 945-954 (2005)

C. Pinte, W.R.F. Dent, F. Ménard, A. Hales, T. Hill, P. Cortes, I. de Gregorio-Monsalvo, Astrophys. J. 816, 25 (2016)

K.M. Pontoppidan, S.M. Blevins, Faraday Discuss. 169, 49-60 (2014)

K.M. Pontoppidan, G.A. Blake, A. Smette, Astrophys. J. 733, 84 (2011)

S.H. Pravdo, E.D. Feigelson, G. Garmire, Y. Maeda, Y. Tsuboi, J. Bally, Nature 413, 708-711 (2001)

L. Prisinzano, G. Micela, E. Flaccomio, J.R. Stauffer, T. Megeath, L. Rebull, M. Robberto, K. Smith, E.D. Feigelson, N. Grosso, S. Wolk, Astrophys. J. 677, 401-424 (2008)

K.M. Punzi, P. Hily-Blant, J.H. Kastner, G.G. Sacco, T. Forveille, Astrophys. J. 805, 147 (2015)

C. Qi, K.I. Öberg, D.J. Wilner, P. D’Alessio, E. Bergin, S.M. Andrews, G.A. Blake, M.R. Hogerheijde, E.F. van Dishoeck, Science 341, 630-632 (2013)

C. Qi, K.I. Öberg, S.M. Andrews, D.J. Wilner, E.A. Bergin, A.M. Hughes, M. Hogherheijde, P. D’Alessio, Astrophys. J. 813, 128 (2015)

R.R. Rafikov, Astrophys. J. Lett. 621, 69-72 (2005)

S.N. Raymond, E. Kokubo, A. Morbidelli, R. Morishima, K.J. Walsh, in Protostars and Planets VI (2014), pp. 595-618

L. Reboussin, V. Wakelam, S. Guilloteau, F. Hersant, A. Dutrey, Astron. Astrophys. 579, 82 (2015)

Z. Regály, A. Juhász, Z. Sándor, C.P. Dullemond, Mon. Not. R. Astron. Soc. 419, 1701-1712 (2012)

W.K.M. Rice, G. Lodato, J.E. Pringle, P.J. Armitage, I.A. Bonnell, Mon. Not. R. Astron. Soc. 355, $543-552$ (2004)

E. Rigliaco, I. Pascucci, U. Gorti, S. Edwards, D. Hollenbach, Astrophys. J. 772, 60 (2013)

P.D. Rogers, J. Wadsley, Mon. Not. R. Astron. Soc. 423, 1896-1908 (2012)

G.G. Sacco, E. Flaccomio, I. Pascucci, F. Lahuis, B. Ercolano, J.H. Kastner, G. Micela, B. Stelzer, M. Sterzik, Astrophys. J. 747, 142 (2012) 
S. Sallum, K.B. Follette, J.A. Eisner, L.M. Close, P. Hinz, K. Kratter, J. Males, A. Skemer, B. Macintosh, P. Tuthill, V. Bailey, D. Defrère, K. Morzinski, T. Rodigas, E. Spalding, A. Vaz, A.J. Weinberger, Nature 527, 342-344 (2015)

C. Salyk, K. Pontoppidan, S. Corder, D. Muñoz, K. Zhang, G.A. Blake, Astrophys. J. 792, 68 (2014)

K.R. Schwarz, E.A. Bergin, L.I. Cleeves, G.A. Blake, K. Zhang, K.I. Öberg, E.F. van Dishoeck, C. Qi, Astrophys. J. 823, 91 (2016)

D. Semenov, D. Wiebe, Astrophys. J. Suppl. Ser. 196, 25 (2011)

D. Semenov, D. Wiebe, T. Henning, Astron. Astrophys. 417, 93-106 (2004)

D. Semenov, D. Wiebe, T. Henning, Astrophys. J. Lett. 647, 57-60 (2006)

F.H. Shu, Astrophys. J. 214, 488-497 (1977)

L. Spitzer Jr., M.G. Tomasko, Astrophys. J. 152, 971 (1968)

D. Stamatellos, A.P. Whitworth, Mon. Not. R. Astron. Soc. 392, 413-427 (2009)

D. Stamatellos, A.P. Whitworth, D.A. Hubber, Astrophys. J. 730, 32 (2011)

B. Stelzer, E. Flaccomio, K. Briggs, G. Micela, L. Scelsi, M. Audard, I. Pillitteri, M. Güdel, Astron. Astrophys. 468, 463-475 (2007)

S. Takakuwa, M. Saito, J. Lim, K. Saigo, T.K. Sridharan, N.A. Patel, Astrophys. J. 754, 52 (2012)

M. Takami, A. Chrysostomou, T.P. Ray, C. Davis, W.R.F. Dent, J. Bailey, M. Tamura, H. Terada, Astron. Astrophys. 416, 213-219 (2004)

R. Teague, D. Semenov, S. Guilloteau, T. Henning, A. Dutrey, V. Wakelam, E. Chapillon, V. Pietu, Astron. Astrophys. 574, 137 (2015)

A. Telleschi, M. Güdel, K.R. Briggs, M. Audard, F. Palla, Astron. Astrophys. 468, 425-442 (2007)

L. Testi, T. Birnstiel, L. Ricci, S. Andrews, J. Blum, J. Carpenter, C. Dominik, A. Isella, A. Natta, J.P. Williams, D.J. Wilner, in Protostars and Planets VI (2014), pp. 339-361

W.-F. Thi, P. Woitke, I. Kamp, Mon. Not. R. Astron. Soc. 412, 711-726 (2011)

I. Tilling, P. Woitke, G. Meeus, A. Mora, B. Montesinos, P. Riviere-Marichalar, C. Eiroa, W.-F. Thi, A. Isella, A. Roberge, C. Martin-Zaidi, I. Kamp, C. Pinte, G. Sandell, W.D. Vacca, F. Ménard, I. Mendigutía, G. Duchêne, W.R.F. Dent, G. Aresu, R. Meijerink, M. Spaans, Astron. Astrophys. 538, 20 (2012)

J.J. Tobin, L. Hartmann, H.-F. Chiang, D.J. Wilner, L.W. Looney, L. Loinard, N. Calvet, P. D’ Alessio, Nature 492, 83-85 (2012)

J.J. Tobin, L. Hartmann, H.-F. Chiang, D.J. Wilner, L.W. Looney, L. Loinard, N. Calvet, P. D’Alessio, Astrophys. J. 771, 48 (2013)

M. Tsujimoto, E.D. Feigelson, N. Grosso, G. Micela, Y. Tsuboi, F. Favata, H. Shang, J.H. Kastner, Astrophys. J. Suppl. Ser. 160, 503-510 (2005)

N.J. Turner, S. Fromang, C. Gammie, H. Klahr, G. Lesur, M. Wardle, X.-N. Bai, in Protostars and Planets $V I$ (2014), pp. 411-432

R. van Boekel, M. Güdel, T. Henning, F. Lahuis, E. Pantin, Astron. Astrophys. 497, 137-144 (2009)

N. van der Marel, E.F. van Dishoeck, S. Bruderer, T. Birnstiel, P. Pinilla, C.P. Dullemond, T.A. van Kempen, M. Schmalzl, J.M. Brown, G.J. Herczeg, G.S. Mathews, V. Geers, Science 340, 1199-1202 (2013)

N. van der Marel, P. Pinilla, J. Tobin, T. van Kempen, S. Andrews, L. Ricci, T. Birnstiel, Astrophys. J. Lett. 810, 7 (2015)

G. van der Plas, S. Casassus, F. Ménard, S. Perez, W.F. Thi, C. Pinte, V. Christiaens, Astrophys. J. Lett. 792, 25 (2014)

A.I. Vasyunin, D.S. Wiebe, T. Birnstiel, S. Zhukovska, T. Henning, C.P. Dullemond, Astrophys. J. 727, 76 (2011)

R. Visser, E.A. Bergin, Astrophys. J. Lett. 754, 18 (2012)

R. Visser, E.A. Bergin, J.K. Jørgensen, Astron. Astrophys. 577, 102 (2015)

R. Visser, E.F. van Dishoeck, J.H. Black, Astron. Astrophys. 503, 323-343 (2009)

E.I. Vorobyov, Astrophys. J. 723, 1294-1307 (2010)

E.I. Vorobyov, Astrophys. J. Lett. 728, 45 (2011a)

E.I. Vorobyov, Astrophys. J. 729, 146 (2011b)

E.I. Vorobyov, Astron. Astrophys. 552, 129 (2013)

E.I. Vorobyov, Astron. Astrophys. 590, A115 (2016). doi:10.1051/0004-6361/201628102

E.I. Vorobyov, S. Basu, Astrophys. J. 650, 956-969 (2006)

E.I. Vorobyov, S. Basu, Astrophys. J. Lett. 714, 133-137 (2010a)

E.I. Vorobyov, S. Basu, Astrophys. J. 719, 1896-1911 (2010b)

E.I. Vorobyov, S. Basu, Astrophys. J. 805, 115 (2015)

E.I. Vorobyov, I. Baraffe, T. Harries, G. Chabrier, Astron. Astrophys. 557, 35 (2013)

V. Wakelam, J.-C. Loison, E. Herbst, B. Pavone, A. Bergeat, K. Béroff, M. Chabot, A. Faure, D. Galli, W.D. Geppert, D. Gerlich, P. Gratier, N. Harada, K.M. Hickson, P. Honvault, S.J. Klippenstein, S.D. Le Picard, G. Nyman, M. Ruaud, S. Schlemmer, I.R. Sims, D. Talbi, J. Tennyson, R. Wester, Astrophys. J. Suppl. Ser. 217, 20 (2015) 
C. Walsh, T.J. Millar, H. Nomura, Astrophys. J. 722, 1607-1623 (2010)

C. Walsh, H. Nomura, T.J. Millar, Y. Aikawa, Astrophys. J. 747, 114 (2012)

C. Walsh, E. Herbst, H. Nomura, T.J. Millar, S.W. Weaver, Faraday Discuss. 168, 389 (2014)

C. Walsh, R.A. Loomis, K.I. Öberg, M. Kama, M.L.R. van 't Hoff, T.J. Millar, Y. Aikawa, E. Herbst, S.L.W. Weaver, H. Nomura, Astrophys. J. Lett. 823(1), 10 (2016). http://stacks.iop.org/ $2041-8205 / 823 / \mathrm{i}=1 / \mathrm{a}=\mathrm{L} 10$

J.P. Williams, W.M.J. Best, Astrophys. J. 788, 59 (2014)

J.P. Williams, L.A. Cieza, Annu. Rev. Astron. Astrophys. 49, 67-117 (2011)

P. Woitke, I. Kamp, W.-F. Thi, Astron. Astrophys. 501, 383-406 (2009)

P. Woitke, M. Min, C. Pinte, W.-F. Thi, I. Kamp, C. Rab, F. Anthonioz, S. Antonellini, C. Baldovin-Saavedra, A. Carmona, C. Dominik, O. Dionatos, J. Greaves, M. Güdel, J.D. Ilee, A. Liebhart, F. Ménard, L. Rigon, L.B.F.M. Waters, G. Aresu, R. Meijerink, M. Spaans, Astron. Astrophys. 586, 103 (2016)

K. Zhang, G.A. Blake, E.A. Bergin, Astrophys. J. Lett. 806, 7 (2015)

Z. Zhu, L. Hartmann, N. Calvet, J. Hernandez, J. Muzerolle, A.-K. Tannirkulam, Astrophys. J. 669, $483-492$ (2007)

Z. Zhu, L. Hartmann, R.P. Nelson, C.F. Gammie, Astrophys. J. 746, 110 (2012) 